\title{
Spectroscopic Investigations and DFT Calculations on 3-(Diacetylamino)-2-ethyl-3H-quinazolin-4-one
}

\author{
Yusuf Sert, ${ }^{1,2}$ Fatih Ucun, ${ }^{3}$ Gamal A. El-Hiti, ${ }^{4}$ Keith Smith, ${ }^{5}$ and Amany S. Hegazy ${ }^{5}$ \\ ${ }^{1}$ Department of Physics, Faculty of Art \& Sciences, Bozok University, 66100 Yozgat, Turkey \\ ${ }^{2}$ Sorgun Vocational School, Bozok University, 66100 Yozgat, Turkey \\ ${ }^{3}$ Department of Physics, Faculty of Art \& Sciences, Süleyman Demirel University, 32100 Isparta, Turkey \\ ${ }^{4}$ Cornea Research Chair, Department of Optometry, College of Applied Medical Sciences, King Saud University, \\ P.O. Box 10219, Riyadh 11433, Saudi Arabia \\ ${ }^{5}$ School of Chemistry, Cardiff University, Main Building, Park Place, Cardiff CF10 3AT, UK \\ Correspondence should be addressed to Gamal A. El-Hiti; gelhiti@ksu.edu.sa
}

Received 23 October 2015; Accepted 28 January 2016

Academic Editor: Christoph Krafft

Copyright ( 2016 Yusuf Sert et al. This is an open access article distributed under the Creative Commons Attribution License, which permits unrestricted use, distribution, and reproduction in any medium, provided the original work is properly cited.

\begin{abstract}
The theoretical and experimental vibrational frequencies of 3-(diacetylamino)-2-ethyl-3H-quinazolin-4-one (2) were investigated. The experimental Laser-Raman spectrum $\left(4000-100 \mathrm{~cm}^{-1}\right)$ and FT-IR spectrum $\left(4000-400 \mathrm{~cm}^{-1}\right)$ of the newly synthesized compound were recorded in the solid phase. Both the theoretical vibrational frequencies and the optimized geometric parameters such as bond lengths and bond angles have for the first time been calculated using density functional theory (DFT/B3LYP and DFT/M06-2X) quantum chemical methods with the 6-311++G(d,p) basis set using Gaussian 03 software. The vibrational frequencies were assigned with the help of potential energy distribution (PED) analysis using VEDA 4 software. The calculated vibrational frequencies and the optimized geometric parameters were found to be in good agreement with the corresponding reported experimental data. Also, the energies of the lowest unoccupied molecular orbital (LUMO), highest occupied molecular orbital (HOMO), and other related molecular energies for 3-(diacetylamino)-2-ethyl-3H-quinazolin-4-one (2) have been investigated using the same computational methods.
\end{abstract}

\section{Introduction}

Quinazoline derivatives are of interest because they show a variety of biological activities including anti-inflammation [1], antibacterial [2], antispasm [3], anticancer [4, 5], antiobesity [6] and reductase inhibitory properties [7]. Quinazolin4-one derivatives have been synthesized by various methods, for example, reactions of carbon dioxide with 2aminobenzonitrile without use of a catalyst in water [8]; reactions of $\mathrm{N}$-substituted 2-bromobenzamides with formamide catalysed by CuI and 4-hydroxy-l-proline [9]; threecomponent reactions of benzyl halides, isatoic anhydride, and primary amines under mild Kornblum conditions [10]. We have shown that the quinazoline ring system can be easily modified via lithiation by a lithium reagent such as alkyllithium in anhydrous tetrahydrofuran at low temperature followed by reactions with electrophiles to provide access to substituted derivatives in high yields. Such derivatives might be difficult to synthesize by other means [11-15].

As far as we are aware, there have been no previous reports of quantum chemical calculations or FT-IR and Laser-Raman spectral studies on 3-(diacetylamino)-2-ethyl$3 H$-quinazolin-4-one (2). Herein, we report experimental infrared and Raman spectra along with quantum chemical calculations, which correlate well with each other, to enable vibrational frequencies for compound $\mathbf{2}$ to be assigned.

\section{Experimental Details}

2.1. Characterization Techniques. FT-IR spectra over the range $4000-400 \mathrm{~cm}^{-1}$ were obtained on solid phase samples at room temperature using a Perkin-Elmer Spectrum Two FT-IR Spectrometer with $4 \mathrm{~cm}^{-1}$ resolution. Raman spectra within the range of $4000-100 \mathrm{~cm}^{-1}$ were obtained on solid 
phase samples using a Renishaw inVia Raman microscope (excitation line at $785 \mathrm{~nm}$ from a diode laser; 100 scans, resolution $1 \mathrm{~cm}^{-1}$ ).

Melting point determination was performed on a Gallenkamp melting point apparatus by the open capillary method. ${ }^{1} \mathrm{H}(500 \mathrm{MHz})$ and ${ }^{13} \mathrm{C}$ NMR $(125 \mathrm{MHz})$ spectra were recorded on a Bruker AV500 spectrometer in deuterated dimethyl sulfoxide (DMSO- $d_{6}$ ). Chemical shifts $\delta(\mathrm{ppm})$ are reported relative to tetramethylsilane (TMS) and coupling constants $(J)$ are in Hz. DEPT spectra were used to detect the ${ }^{13} \mathrm{C}$ multiplicities. Coupling patterns, integration values, and expected chemical shifts were used to assign signals. The low and high-resolution mass spectra were recorded on Waters GCT Premier and Waters LCT Premier XE instruments, respectively. A Nonius Kappa CCD diffractometer was used to record the X-ray single-crystal diffraction data by the use of graphite-monochromated Mo- $\mathrm{K}_{\alpha},(\lambda=0.71073 \AA)$ radiation. The structure was solved by direct methods using SHELXS96 [16] and refined with all data on $F^{2}$ full-matrix least squares using SHELXL-97 [17]. The full crystallographic data (CCDC 971829) for the title compound can be obtained via http://www.ccdc.cam.ac.uk/structures.

2.2. Chemicals. Chemicals and reagents from Sigma-Aldrich were used without further purification and Fischer Scientific silica $60 \mathrm{~A}$ (35-70 micron) was used in the purification of $\mathbf{2}$ by column chromatography.

2.3. Synthesis of 3-(Diacetylamino)-2-ethyl-3H-quinazolin-4one (2). Acetic anhydride $\left(\mathrm{Ac}_{2} \mathrm{O} ; 2.1 \mathrm{~mL}, 22.0 \mathrm{mmol}\right)$ in anhydrous toluene $(5 \mathrm{~mL})$ was added to a stirred mixture of 3-amino-2-ethyl-3H-quinazolin-4-one (1; $1.89 \mathrm{~g}, 10.0 \mathrm{mmol})$ and triethylamine (TEA; $5 \mathrm{~mL}$ ) in dry toluene $(20 \mathrm{~mL}$ ) (Scheme 1). The mixture was refluxed for $30 \mathrm{~min}$, allowed to cool, washed twice with saturated aq. $\mathrm{NaHCO}_{3}(10 \mathrm{~mL})$ and water $(15 \mathrm{~mL})$, and dried $\left(\mathrm{MgSO}_{4}\right)$, and the solvent was evaporated under vacuum. Column chromatography (silica; $\mathrm{Et}_{2} \mathrm{O}$-hexane, 1:4) was used to purify the crude product to give a white powder. Crystallization from ethyl acetate provided colorless crystals of 2 ( $2.58 \mathrm{~g}, 9.4 \mathrm{mmol}$; 94\%); m.p. $110-111^{\circ} \mathrm{C} .{ }^{1} \mathrm{H}$ NMR $\left(500 \mathrm{MHz}, \mathrm{DMSO}-d_{6}, \delta, \mathrm{ppm}\right): 8.16$ (dd, $J=8.0,1.2 \mathrm{~Hz}, 1 \mathrm{H}, \mathrm{H}-5$ ), 7.94 (app. dt, $J=1.2,8.0 \mathrm{~Hz}, 1 \mathrm{H}, \mathrm{H}-$ 7), 7.76 (br app. d, $J=8.0 \mathrm{~Hz}, 1 \mathrm{H}, \mathrm{H}-8$ ), 7.61 (app. dt, $J=1.2$, $8.0 \mathrm{~Hz}, 1 \mathrm{H}, \mathrm{H}-6), 2.67\left(\mathrm{q}, J=7.2 \mathrm{~Hz}, 2 \mathrm{H}, \mathrm{CH}_{2}\right.$ ), 2.40 (s, 6 $\left.\mathrm{H}, 2 \mathrm{CH}_{3} \mathrm{C}=\mathrm{O}\right), 1.26\left(\mathrm{t}, J=7.2 \mathrm{~Hz}, 3 \mathrm{H}, \mathrm{CH}_{3} \mathrm{CH}_{2}\right) \cdot{ }^{13} \mathrm{C} \mathrm{NMR}$ $\left(125 \mathrm{MHz}, \mathrm{DMSO}-d_{6}, \delta, \mathrm{ppm}\right): 170.8(\mathrm{~s}, 2 \mathrm{C}=\mathrm{O}), 159.4$ (s, C4), 158.0 (s, C-2), 146.9 (s, C-8a), 136.2 (d, C-7), 128.0 (d, C5), 127.8 (d, C-6), 127.2 (d, C-8), 120.7 (s, C-4a), $25.7\left(\mathrm{t}, \mathrm{CH}_{2}\right.$ ), 24.9 (q, $\left.2 \mathrm{CH}_{3} \mathrm{C}=\mathrm{O}\right), 10.4\left(\mathrm{q}, \mathrm{CH}_{3} \mathrm{CH}_{2}\right)$. CI-MS: $(\mathrm{m} / z, \%):=$ $274\left([M H]^{+}, 100\right)$. HRMS (CI): Calculated for $\mathrm{C}_{14} \mathrm{H}_{16} \mathrm{~N}_{3} \mathrm{O}_{3}$ $[M H]^{+}$274.1192; found: 274.1201 .

\section{Computational Details}

The use of density functional theory (DFT) calculations has increased rapidly for various applications, particularly since accurate nonlocal corrections were introduced. The methods
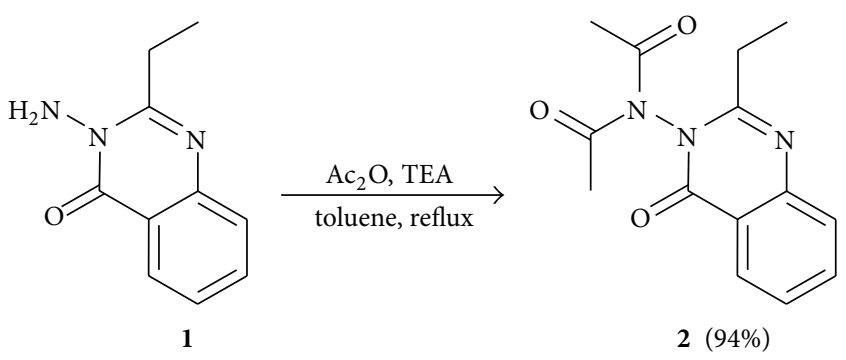

SCHEme 1: Synthesis of 3-(diacetylamino)-2-ethyl-3H-quinazolin-4one (2).

employed in the current work have been used in many previous theoretical studies [18-32].

Initial atomic coordinates can generally be taken from experimental XRD results or a database. In this work, experimental XRD data and the Gauss View software database have each been used to determine initial atomic coordinates and to optimize the input structure. The most stable structure after optimization was obtained from the initial atomic coordinates taken from the Gauss View database [33]. This most stable structure, following optimization, was used for further theoretical analysis. The calculated gas phase ground state molecular structure of the title compound was optimized by the use of DFT/B3LYP and DFT/M06-2X methods with the 6-311++G(d,p) basis set, and the calculated optimized structure was used in the vibrational frequency calculations. The calculated harmonic vibrational frequencies were scaled by 0.9614 (B3LYP) and 0.9489 (M06-2X) for use with the $6-311++G(d, p)$ basis set, respectively $[33,34]$. Identical scale factors were used for the entire spectra. The Gauss View molecular visualization program [33] and the Gaussian 03 program [35] were used to calculate vibrational wavenumbers, optimized geometric parameters, and other molecular properties. The calculated vibrational frequencies were assigned via potential energy distribution (PED) analysis of all the fundamental vibration modes by the use of the VEDA 4 program $[36,37$ that has been used previously $[20,23,24,32,38,39]$. All the vibrational assignments were based on the B3LYP/6-311++G(d,p) level calculations. Consequently, some assignments could correspond to the value of the next or previous vibrational frequency at the M06-2X/6-311++G(d,p) level.

\section{Results and Discussion}

4.1. Synthesis. 3-(Diacetylamino)-2-ethyl-3H-quinazolin-4one (2) was synthesized, in $94 \%$ yield after crystallization, by double acetylation of 3-amino-2-ethyl-3H-quinazolin-4-one (1) with acetic anhydride (two mole equivalents) in boiling toluene for $3 \mathrm{~h}$ in the presence of triethylamine (Scheme 1). Various spectroscopic techniques were used to confirm the structure of 2.

4.2. Geometric Structure. The single-crystal $\mathrm{X}$-ray crystallographic analysis of $2\left(\mathrm{C}_{14} \mathrm{H}_{15} \mathrm{~N}_{3} \mathrm{O}_{3}\right)$ showed that the crystal belonged to the monoclinic system and the $P 2_{1} / c$ space group and possessed the following cell dimensions: $a=7.4975 \AA$, 


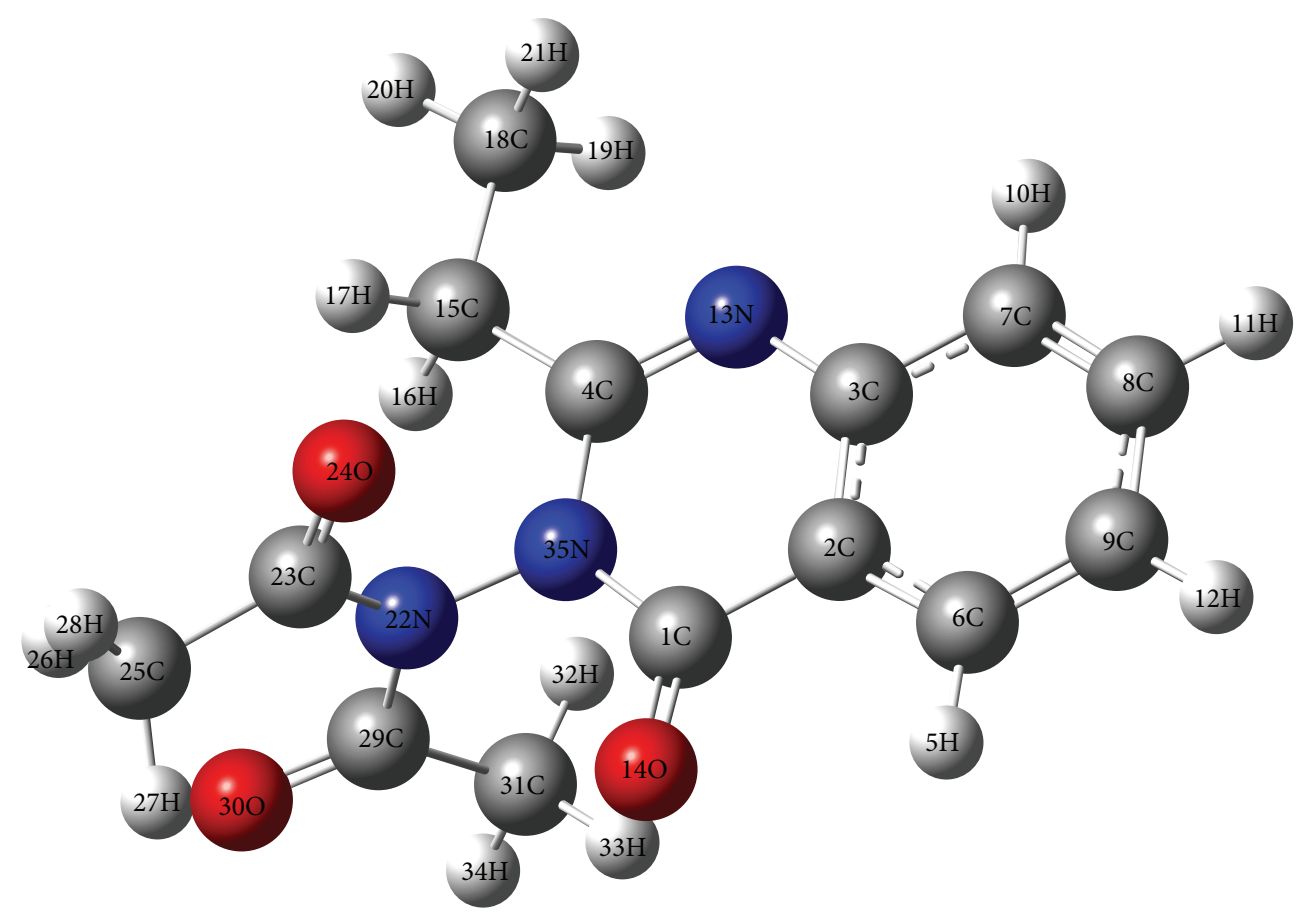

FIgURE 1: The optimized molecular structure of compound 2.

$b=12.0246 \AA$, and $c=15.1939 \AA$ and $\alpha=90^{\circ}, \beta=$ $97.399^{\circ}, \gamma=90^{\circ}$, and $V=1358.39 \AA^{3}$. The measured density of compound 2 was $1.336 \mathrm{mg} / \mathrm{mm}^{3}$. Table 1 shows the bond lengths and bond angles for the optimized theoretical and experimental structures along with the atom numbering scheme (Figure 1).

As can be seen from Table 1, the optimized parameters calculated at both DFT levels differ only slightly from the experimental values, and small variations are to be expected since the calculations correspond to the gas phase rather than the solid state. For example, in the quinazoline moiety, the $\mathrm{C} 4-\mathrm{N} 13$ and $\mathrm{C} 4-\mathrm{N} 35$ bond lengths are calculated as 1.286 and $1.401 \AA$, respectively, by the B3LYP method, and 1.280 and $1.397 \AA$, respectively, by the M06-2X method, while the experimental values are 1.286 and $1.400 \AA$, respectively. Similarly, the N13-C4-N35 and C4-N35-C1 bond angles were calculated as 121.9 and $123.6^{\circ}$, respectively, by the B3LYP method and 122.3 and $123.9^{\circ}$, respectively, by the M06-2X method, while the experimental bond angles were 121.7 and $124.5^{\circ}$, respectively.

In order to quantify the level of agreement between the experimental and computational results, correlation coefficients $\left(R^{2}\right)$ for linear regression analysis of the experimental and theoretical bond angles and lengths were calculated (last row in each section of Table 1 ). The values are $0.9820 / 0.9799$ for bond lengths and $0.9596 / 0.9599$ for bond angles for B3LYP/M06-2X. These values show that bond lengths calculated using the B3LYP method are slightly closer to the experimental data, but bond angles calculated using the M06-2X method are marginally closer to the data obtained experimentally.
The largest variations between the experimental and calculated (B3LYP/6-311++G(d,p)) bond lengths are $0.016 \AA$ for the C1-N35 bond; $0.011 \AA$ for the $\mathrm{C} 3-\mathrm{N} 13$ bond; $0.018 \AA$ for the $\mathrm{C} 8-\mathrm{C} 9$ bond; $0.013 \AA$ for the $\mathrm{C} 15-\mathrm{C} 18$ and $\mathrm{C} 23-\mathrm{C} 25$ bonds; and $0.015 \AA$ for the $\mathrm{C} 29-\mathrm{C} 31$ bond. For bond angles the largest differences are $0.8^{\circ}$ for $\mathrm{N} 13-\mathrm{C} 4-\mathrm{C} 15 ; 0.9^{\circ}$ for $\mathrm{C} 23-$ N22-C29, C29-N22-N35, and C1-N35-C4; and 0.6 for C4$\mathrm{N} 35-\mathrm{N} 22$.

The values found or calculated for $\mathbf{2}$ are consistent with values found experimentally or computationally for corresponding bonds and angles in related compounds such as 2ethyl-3H-quinazoline-4-thione (experimental values available [40]), 3-amino-2-phenyl-3H-quinazolin-4-one (values calculated using the B3LYP/6-31G*, B3LYP/6-311++G(d,p), and M06-2X methods available [41]), 3-(4-methoxybenzylideneamino)-2-phenyl-3H-quinazolin-4-one (values calculated using the B3LYP/6-31G* basis set available [42]), methyl 3,5dibromo-2-diacetylaminobenzoate (experimental data available [43]), and $\mathrm{N}$-(1-diacetylamino- $\mathrm{H}$-tetrazol-5-yl)acetamide (experimental data available [44]).

4.3. Vibrational Analysis. As seen from Figure 1, the molecule has 35 atoms, so there are 105 motions, 3 of which are translational, 3 of which are rotational, and 99 of which are vibration modes. The molecule has $\mathrm{C}_{1}$ symmetry. The experimental FT-IR and Laser-Raman spectra of compound $\mathbf{2}$ are compared with the selected theoretical spectra in Figures 2 and 3, respectively (identified bands are those discussed in the text and bold numbers in Table 2). The observed vibrational frequencies, scaled harmonic vibrational frequencies calculated at both B3LYP and M06-2X levels, and 
TABLE 1: Experimental and calculated geometric parameters of compound 2 (figures in bold relate to parameters discussed in the text).

\begin{tabular}{|c|c|c|c|}
\hline \multirow{2}{*}{ Geometric parameters } & \multirow{2}{*}{ Experimental values } & \multicolumn{2}{|c|}{ Calculated values } \\
\hline & & B3LYP/6-311++G(d,p) & $\mathrm{M} 06-2 \mathrm{X} / 6-311++\mathrm{G}(\mathrm{d}, \mathrm{p})$ \\
\hline \multicolumn{4}{|l|}{ Bond lengths $(\AA)$} \\
\hline $\mathrm{C} 1-\mathrm{C} 2$ & 1.458 & 1.461 & 1.462 \\
\hline $\mathrm{C} 1-\mathrm{O} 14$ & 1.216 & 1.216 & 1.209 \\
\hline C1-N35 & 1.406 & 1.422 & 1.411 \\
\hline $\mathrm{C} 2-\mathrm{C} 3$ & 1.399 & 1.409 & 1.402 \\
\hline $\mathrm{C} 2-\mathrm{C} 6$ & 1.403 & 1.403 & 1.399 \\
\hline $\mathrm{C} 3-\mathrm{C} 7$ & 1.402 & 1.407 & 1.403 \\
\hline $\mathrm{C} 3-\mathrm{N} 13$ & 1.398 & 1.387 & 1.389 \\
\hline $\mathrm{C} 4-\mathrm{N} 13$ & 1.286 & 1.286 & 1.280 \\
\hline $\mathrm{C} 4-\mathrm{C} 15$ & 1.499 & 1.509 & 1.505 \\
\hline $\mathrm{C} 4-\mathrm{N} 35$ & 1.400 & 1.401 & 1.397 \\
\hline H5-C6 & 0.93 & 1.083 & 1.084 \\
\hline C6-C9 & 1.375 & 1.383 & 1.380 \\
\hline $\mathrm{C} 7-\mathrm{C} 8$ & 1.372 & 1.384 & 1.381 \\
\hline $\mathrm{C} 7-\mathrm{H} 10$ & 0.93 & 1.083 & 1.083 \\
\hline $\mathrm{C} 8-\mathrm{C} 9$ & 1.387 & 1.405 & 1.403 \\
\hline C8-H11 & 0.93 & 1.084 & 1.084 \\
\hline C9-H12 & 0.93 & 1.084 & 1.083 \\
\hline C15-H16 & 0.97 & 1.096 & 1.095 \\
\hline $\mathrm{C} 15-\mathrm{H} 17$ & 0.97 & 1.095 & 1.095 \\
\hline C15-C18 & 1.515 & 1.528 & 1.522 \\
\hline C18-H19 & 0.96 & 1.091 & 1.090 \\
\hline $\mathrm{C} 18-\mathrm{H} 20$ & 0.96 & 1.093 & 1.091 \\
\hline $\mathrm{C} 18-\mathrm{H} 21$ & 0.96 & 1.090 & 1.089 \\
\hline $\mathrm{N} 22-\mathrm{C} 23$ & 1.422 & 1.442 & 1.433 \\
\hline N22-C29 & 1.417 & 1.421 & 1.416 \\
\hline N22-N35 & 1.401 & 1.395 & 1.383 \\
\hline $\mathrm{C} 23-\mathrm{O} 24$ & 1.201 & 1.207 & 1.201 \\
\hline $\mathrm{C} 23-\mathrm{C} 25$ & 1.490 & 1.503 & 1.499 \\
\hline $\mathrm{C} 25-\mathrm{H} 26$ & 0.96 & 1.091 & 1.089 \\
\hline $\mathrm{C} 25-\mathrm{H} 27$ & 0.96 & 1.090 & 1.089 \\
\hline $\mathrm{C} 25-\mathrm{H} 28$ & 0.96 & 1.089 & 1.088 \\
\hline $\mathrm{C} 29-\mathrm{O} 30$ & 1.201 & 1.208 & 1.201 \\
\hline C29-C31 & 1.496 & 1.511 & 1.508 \\
\hline C31-H32 & 0.96 & 1.092 & 1.092 \\
\hline C31-H33 & 0.96 & 1.092 & 1.092 \\
\hline C31-H34 & 0.96 & 1.088 & 1.087 \\
\hline$R^{2}$ & & 0.982 & 0.9799 \\
\hline \multicolumn{4}{|l|}{ Bond angles $\left({ }^{\circ}\right)$} \\
\hline $\mathrm{C} 2-\mathrm{C} 1-\mathrm{O} 14$ & 126.6 & 126.5 & 126.6 \\
\hline C2-C1-N35 & 112.9 & 113.1 & 112.8 \\
\hline O14-C1-N35 & 120.5 & 120.4 & 120.5 \\
\hline $\mathrm{C} 1-\mathrm{C} 2-\mathrm{C} 3$ & 119.3 & 119.3 & 119.3 \\
\hline $\mathrm{C} 1-\mathrm{C} 2-\mathrm{C} 6$ & 120.2 & 120.1 & 119.8 \\
\hline $\mathrm{C} 3-\mathrm{C} 2-\mathrm{C} 6$ & 120.4 & 120.6 & 120.9 \\
\hline $\mathrm{C} 2-\mathrm{C} 3-\mathrm{C} 7$ & 118.9 & 118.8 & 118.7 \\
\hline $\mathrm{C} 2-\mathrm{C} 3-\mathrm{N} 13$ & 122.9 & 122.6 & 122.8 \\
\hline C7-C3-N13 & 118.1 & 118.6 & 118.4 \\
\hline
\end{tabular}


TABle 1: Continued.

\begin{tabular}{|c|c|c|c|}
\hline \multirow{2}{*}{ Geometric parameters } & \multirow{2}{*}{ Experimental values } & \multicolumn{2}{|c|}{ Calculated values } \\
\hline & & B3LYP/6-311++G(d,p) & M06-2X/6-311++G(d,p) \\
\hline N13-C4-C15 & 121.5 & 120.7 & 121.1 \\
\hline N13-C4-N35 & 121.7 & 121.9 & 122.3 \\
\hline C15-C4-N35 & 116.8 & 117.3 & 116.5 \\
\hline C2-C6-H5 & 120.3 & 118.6 & 118.5 \\
\hline $\mathrm{C} 2-\mathrm{C} 6-\mathrm{C} 9$ & 119.5 & 119.8 & 119.6 \\
\hline $\mathrm{H} 5-\mathrm{C} 6-\mathrm{C} 9$ & 119.9 & 121.6 & 121.9 \\
\hline $\mathrm{C} 3-\mathrm{C} 7-\mathrm{C} 8$ & 119.8 & 120.1 & 120.1 \\
\hline $\mathrm{C} 3-\mathrm{C} 7-\mathrm{H} 10$ & 120.1 & 118.3 & 118.1 \\
\hline $\mathrm{C} 8-\mathrm{C} 7-\mathrm{H} 10$ & 120.1 & 121.6 & 121.8 \\
\hline $\mathrm{C} 7-\mathrm{C} 8-\mathrm{C} 9$ & 121.2 & 120.7 & 120.9 \\
\hline C7-C8-H11 & 119.4 & 119.6 & 119.6 \\
\hline C9-C8-H11 & 119.9 & 119.6 & 119.5 \\
\hline C6-C9-C8 & 120.1 & 119.9 & 119.8 \\
\hline C6-C9-H12 & 119.9 & 120.2 & 120.2 \\
\hline C8-C9-H12 & 119.9 & 119.9 & 119.9 \\
\hline $\mathrm{C} 3-\mathrm{N} 13-\mathrm{C} 4$ & 121.7 & 119.4 & 118.7 \\
\hline $\mathrm{C} 4-\mathrm{C} 15-\mathrm{H} 16$ & 108.9 & 109.0 & 108.8 \\
\hline C4-C15-H17 & 108.9 & 107.8 & 107.5 \\
\hline $\mathrm{C} 4-\mathrm{C} 15-\mathrm{C} 18$ & 113.3 & 113.7 & 112.7 \\
\hline $\mathrm{H} 16-\mathrm{C} 15-\mathrm{H} 17$ & 107.7 & 105.7 & 106.1 \\
\hline $\mathrm{H} 16-\mathrm{C} 15-\mathrm{C} 18$ & 108.9 & 110.1 & 110.8 \\
\hline H17-C15-C18 & 108.9 & 110.1 & 110.6 \\
\hline $\mathrm{C} 15-\mathrm{C} 18-\mathrm{H} 19$ & 109.5 & 111.4 & 110.9 \\
\hline $\mathrm{C} 15-\mathrm{C} 18-\mathrm{H} 20$ & 109.5 & 109.5 & 109.7 \\
\hline $\mathrm{C} 15-\mathrm{C} 18-\mathrm{H} 21$ & 109.5 & 111.1 & 110.7 \\
\hline $\mathrm{H} 19-\mathrm{C} 18-\mathrm{H} 20$ & 109.5 & 108.6 & 108.9 \\
\hline $\mathrm{H} 19-\mathrm{C} 18-\mathrm{H} 21$ & 109.5 & 107.5 & 107.6 \\
\hline $\mathrm{H} 20-\mathrm{C} 18-\mathrm{H} 21$ & 109.5 & 108.6 & 108.9 \\
\hline $\mathrm{C} 23-\mathrm{N} 22-\mathrm{C} 29$ & 127.8 & 126.9 & 127.1 \\
\hline $\mathrm{C} 23-\mathrm{N} 22-\mathrm{N} 35$ & 114.0 & 114.1 & 114.0 \\
\hline $\mathrm{C} 29-\mathrm{N} 22-\mathrm{N} 35$ & 117.2 & 118.1 & 117.8 \\
\hline $\mathrm{N} 22-\mathrm{C} 23-\mathrm{O} 24$ & 118.4 & 117.9 & 117.8 \\
\hline N22-C23-C25 & 118.4 & 118.8 & 118.4 \\
\hline $\mathrm{O} 24-\mathrm{C} 23-\mathrm{C} 25$ & 123.3 & 123.4 & 123.8 \\
\hline $\mathrm{C} 23-\mathrm{C} 25-\mathrm{H} 26$ & 109.5 & 111.1 & 110.7 \\
\hline $\mathrm{C} 23-\mathrm{C} 25-\mathrm{H} 27$ & 109.5 & 111.2 & 110.7 \\
\hline $\mathrm{C} 23-\mathrm{C} 25-\mathrm{H} 28$ & 109.5 & 106.9 & 106.7 \\
\hline $\mathrm{H} 26-\mathrm{C} 25-\mathrm{H} 27$ & 109.5 & 106.6 & 106.9 \\
\hline $\mathrm{H} 26-\mathrm{C} 25-\mathrm{H} 28$ & 109.5 & 110.5 & 110.8 \\
\hline $\mathrm{H} 27-\mathrm{C} 25-\mathrm{H} 28$ & 109.5 & 110.7 & 110.9 \\
\hline $\mathrm{N} 22-\mathrm{C} 29-\mathrm{O} 30$ & 120.5 & 120.9 & 121.1 \\
\hline N22-C29-C31 & 116.7 & 116.3 & 115.5 \\
\hline O30-C29-C31 & 122.8 & 122.9 & 123.4 \\
\hline C29-C31-H32 & 109.5 & 112.2 & 111.5 \\
\hline C29-C31-H33 & 109.5 & 110.2 & 109.8 \\
\hline C29-C31-H34 & 109.5 & 107.3 & 107.3 \\
\hline H32-C31-H33 & 109.5 & 108.1 & 108.3 \\
\hline H32-C31-H34 & 109.5 & 109.6 & 110.0 \\
\hline H33-C31-H34 & 109.5 & 109.6 & 109.9 \\
\hline C1-N35-C4 & 124.5 & 123.6 & 123.9 \\
\hline
\end{tabular}


TABle 1: Continued.

\begin{tabular}{lccc}
\hline Geometric parameters & \multicolumn{2}{c}{ Experimental values } & \multicolumn{2}{c}{ Calculated values } \\
& & B3LYP/6-311++G(d,p) & M06-2X/6-311++G(d,p) \\
\hline C1-N35-N22 & 116.1 & 115.9 & 115.9 \\
C4-N35-N22 & 119.2 & 119.8 & 119.4 \\
$R^{2}$ & & $\mathbf{0 . 9 5 9 6}$ & $\mathbf{0 . 9 5 9 9}$ \\
\hline
\end{tabular}

TABLE 2: Observed and calculated vibrational frequencies of title compound with 6-311++G(d,p).

\begin{tabular}{|c|c|c|c|c|c|}
\hline \multirow{2}{*}{ Vibration number } & \multirow{2}{*}{ Assignment $(\mathrm{PED} \%)^{\mathrm{a}}$} & \multicolumn{2}{|c|}{ Observed frequencies } & \multicolumn{2}{|c|}{ Calculated frequencies in $\mathrm{cm}^{-1}$} \\
\hline & & FT-IR & Laser-Raman & B3LYP & M06-2X \\
\hline$v_{1}$ & $v \mathrm{CH}(93)$ in ring 1 & 3083 & 3079 & 3078 & 3057 \\
\hline$v_{2}$ & $v \mathrm{CH}(98)$ in ring 1 & 3083 & 3079 & 3074 & 3052 \\
\hline$v_{3}$ & $v \mathrm{CH}(89)$ in ring 1 & 3083 & 3079 & 3063 & 3042 \\
\hline$v_{4}$ & $v \mathrm{CH}(94)$ in ring 1 & 3045 & 3079 & 3048 & 3028 \\
\hline$v_{5}$ & $v \mathrm{CH}(91)$ in asymm. str. $\mathrm{CH}_{3}$ & 3045 & 3079 & 3033 & 3025 \\
\hline$v_{6}$ & $v \mathrm{CH}(99)$ in asymm. str. $\mathrm{CH}_{3}$ & 3045 & 3079 & 3032 & 3024 \\
\hline$v_{7}$ & $v \mathrm{CH}(100)$ in asymm. str. $\mathrm{CH}_{3}$ & 3003 & 2993 & 3009 & 3006 \\
\hline$v_{8}$ & $v \mathrm{CH}(65)$ in asymm. str. $\mathrm{CH}_{3}$ & 3003 & 2993 & 3003 & 2988 \\
\hline$v_{9}$ & $v \mathrm{CH}(99)$ in asymm. str. $\mathrm{CH}_{3}$ & 3003 & 2993 & 2996 & 2987 \\
\hline$v_{10}$ & $v \mathrm{CH}(99)$ in asymm. str. $\mathrm{CH}_{3}$ & 3003 & 2993 & 2989 & 2977 \\
\hline$v_{11}$ & $v \mathrm{CH}(85)$ in symm. str. $\mathrm{CH}_{3}$ & 2949 & 2938 & 2943 & 2929 \\
\hline$v_{12}$ & $v \mathrm{CH}(92)$ in asymm. str. $\mathrm{CH}_{2}$ & 2934 & 2938 & 2939 & 2922 \\
\hline$v_{13}$ & $v \mathrm{CH}(99)$ in symm. str. $\mathrm{CH}_{3}$ & 2934 & 2938 & 2934 & 2916 \\
\hline$v_{14}$ & $v \mathrm{CH}(98)$ in symm. str. $\mathrm{CH}_{3}$ & 2934 & 2914 & 2928 & 2904 \\
\hline$v_{15}$ & $v \mathrm{CH}(94)$ in symm. str. $\mathrm{CH}_{2}$ & 2915 & 2914 & 2911 & 2888 \\
\hline$v_{16}$ & $v \mathrm{OC}(72)$ in $\mathrm{C} 23-\mathrm{O} 24$ and $\mathrm{C} 29-\mathrm{O} 30$ & 1735 & 1743 & 1720 & 1764 \\
\hline$v_{17}$ & $v \mathrm{OC}(92)$ in $\mathrm{C} 23-\mathrm{O} 24$ and $\mathrm{C} 29-\mathrm{O} 30$ & 1725 & 1725 & 1718 & 1721 \\
\hline$v_{18}$ & $v \mathrm{OC}(72)$ in $\mathrm{O} 14-\mathrm{Cl}$ & 1693 & 1694 & 1683 & 1721 \\
\hline$v_{19}$ & $v \mathrm{NC}(53)$ in $\mathrm{N} 13-\mathrm{C} 4+v \mathrm{CC}(18)$ in ring 1 & 1605 & 1609 & 1593 & 1623 \\
\hline$v_{20}$ & $\begin{array}{l}v \mathrm{CC}(47) \text { in ring } 1+v \mathrm{NC}(14) \text { in } \mathrm{N} 13-\mathrm{C} 4+\delta \mathrm{CCC}(10) \\
\text { in ring } 1\end{array}$ & 1570 & 1578 & 1580 & 1601 \\
\hline$v_{21}$ & $v \mathrm{CC}(53)$ in ring $1+\delta \mathrm{CCC}(30)$ in ring 1 & 1514 & & 1539 & 1559 \\
\hline$v_{22}$ & $\begin{array}{l}\delta \mathrm{HCH}(67) \text { in the } \mathrm{CH}_{3} \text { asym. def. and } \mathrm{CH}_{3} \text { twisting } \\
\text { mode }\end{array}$ & 1442 & 1455 & 1446 & 1445 \\
\hline$v_{23}$ & $\delta \mathrm{HCC}(39)$ in ring $1+\delta \mathrm{CCC}(22)$ in ring 1 & 1442 & 1431 & 1444 & 1436 \\
\hline$v_{24}$ & $\begin{array}{l}\delta \mathrm{HCH}(70) \text { in } \mathrm{CH}_{3} \text { asymm. def. }+\tau \mathrm{HCCN}(14) \text { in } \\
\mathrm{H} 17-\mathrm{C} 15-\mathrm{C} 4-\mathrm{N} 13\end{array}$ & 1426 & 1431 & 1436 & 1425 \\
\hline$v_{25}$ & $v \mathrm{CC}(37)$ in ring $1+\delta \mathrm{HCC}(34)$ in ring 1 & 1426 & 1431 & 1435 & 1417 \\
\hline$v_{26}$ & $\begin{array}{l}\delta \mathrm{HCH}(58) \text { in } \mathrm{CH}_{3} \text { asymm. def. }+\tau \mathrm{HCCN}(13) \text { in } \\
\mathrm{H} 33-\mathrm{C} 31-\mathrm{C} 29-\mathrm{N} 22\end{array}$ & 1426 & 1431 & 1429 & 1415 \\
\hline$v_{27}$ & $\begin{array}{l}\delta \mathrm{HCH}(74) \text { in } \mathrm{CH}_{3} \text { asymm. def. }+\tau \mathrm{HCCN}(13) \text { in } \\
\mathrm{H} 33-\mathrm{C} 31-\mathrm{C} 29-\mathrm{N} 22\end{array}$ & 1409 & 1406 & 1414 & 1405 \\
\hline$v_{28}$ & $\delta \mathrm{HCH}(72)$ in $\mathrm{CH}_{3}$ asymm. def. & 1409 & 1406 & 1413 & 1402 \\
\hline$v_{29}$ & $\delta \mathrm{HCH}(84)$ in $\mathrm{CH}_{2}$ sciss. & 1409 & 1406 & 1406 & 1389 \\
\hline$v_{30}$ & $\delta \mathrm{HCH}(68)$ in $\mathrm{CH}_{3}$ asymm. def. & 1409 & 1382 & 1398 & 1384 \\
\hline$v_{31}$ & $\delta \mathrm{HCH}(79)$ in $\mathrm{CH}_{3}$ symm. Def. & 1364 & 1363 & 1366 & 1353 \\
\hline$v_{32}$ & $\delta \mathrm{HCH}(81)$ in $\mathrm{CH}_{3}$ symm. Def. & 1364 & 1363 & 1349 & 1344 \\
\hline$v_{33}$ & $\delta \mathrm{HCH}(60)$ in $\mathrm{CH}_{3}$ symm. Def. & 1328 & 1333 & 1344 & 1339 \\
\hline$v_{34}$ & $\begin{array}{l}\tau \mathrm{HCCN}(33) \text { in } \mathrm{H} 17-\mathrm{C} 15-\mathrm{C} 4-\mathrm{N} 13 \text { and } \mathrm{CH}_{2} \text { wagging } \\
\text { mode in the ethyl group }\end{array}$ & 1328 & 1333 & 1337 & 1331 \\
\hline
\end{tabular}


TABLE 2: Continued.

\begin{tabular}{|c|c|c|c|c|c|}
\hline \multirow{2}{*}{ Vibration number } & \multirow{2}{*}{ Assignment $(\mathrm{PED} \%)^{\mathrm{a}}$} & \multicolumn{2}{|c|}{ Observed frequencies } & \multicolumn{2}{|c|}{ Calculated frequencies in $\mathrm{cm}^{-1}$} \\
\hline & & FT-IR & Laser-Raman & B3LYP & M06-2X \\
\hline$v_{35}$ & $v \mathrm{CC}(72)$ in ring 1 & 1295 & & 1304 & 1304 \\
\hline$v_{36}$ & $v \mathrm{NN}(12)$ in $\mathrm{N} 22-\mathrm{N} 35$ & 1295 & 1272 & 1272 & 1292 \\
\hline$v_{37}$ & $\begin{array}{l}\delta \mathrm{HCC}(28) \text { in ring } 1+v \mathrm{NC}(11) \text { in } \mathrm{N} 13-\mathrm{C} 3+v \mathrm{CC}(10) \text { in } \\
\text { ring } 1+v \mathrm{NNv}(10) \text { in } \mathrm{N} 22-\mathrm{N} 35\end{array}$ & 1263 & 1272 & 1268 & 1260 \\
\hline$v_{38}$ & $\begin{array}{l}\delta \mathrm{HCC}(63) \text { in } \mathrm{H} 17-\mathrm{C} 15-\mathrm{C} 18 \text { and } \mathrm{H} 21-\mathrm{C} 18-\mathrm{C} 15 \text { and } \\
\text { twisting mode } \mathrm{CH}_{2} \text { in the ethyl group }+\tau \mathrm{HCCN}(14) \text { in } \\
\mathrm{H} 17-\mathrm{C} 15-\mathrm{C} 4-\mathrm{N} 13\end{array}$ & 1240 & 1247 & 1246 & 1246 \\
\hline$v_{39}$ & $v \mathrm{NC}(13)$ in N13-C3 and N22-C29 & 1240 & 1230 & 1235 & 1227 \\
\hline$v_{40}$ & $\begin{array}{l}v \mathrm{CC}(11) \text { in ring } 1+\delta \mathrm{HCC}(10) \text { in ring } 1+\delta \mathrm{CCC}(10) \text { in } \\
\text { ring } 1\end{array}$ & 1214 & 1230 & 1208 & 1218 \\
\hline$v_{41}$ & $v \mathrm{NC}(28)$ in $\mathrm{C} 1-\mathrm{N} 35$ and N22-C23 & 1188 & 1192 & 1190 & 1198 \\
\hline$v_{42}$ & $\begin{array}{l}v \mathrm{NC}(24) \text { in } \mathrm{C} 1-\mathrm{N} 35 \text { and } \mathrm{N} 22-\mathrm{C} 23+\delta \mathrm{HCC}(11) \text { in ring } \\
1\end{array}$ & 1188 & 1192 & 1180 & 1195 \\
\hline$v_{43}$ & $\begin{array}{l}\delta \mathrm{HCC}(10) \text { in ring } 1 \text { and } \mathrm{H} 28-\mathrm{C} 25-\mathrm{C} 23+v \mathrm{NN}(10) \text { in } \\
\mathrm{N} 22-\mathrm{N} 35\end{array}$ & 1149 & 1149 & 1157 & 1168 \\
\hline$v_{44}$ & $\delta \mathrm{HCC}(56)$ in ring 1 & 1110 & 1100 & 1127 & 1109 \\
\hline$v_{45}$ & $\delta \mathrm{HCC}(38)$ in ring $1+\delta \mathrm{CCC}(10)$ in ring 1 & 1100 & 1100 & 1089 & 1082 \\
\hline$v_{46}$ & $v \mathrm{CC}(23)$ in $\mathrm{C} 18-\mathrm{C} 15$ & 1058 & & 1069 & 1076 \\
\hline$v_{47}$ & $\begin{array}{l}\tau \mathrm{HCCN}(43) \text { in } \mathrm{H} 17-\mathrm{C} 15-\mathrm{C} 4-\mathrm{N} 13+\delta \mathrm{HCH}(10) \text { in } \\
\mathrm{H} 17-\mathrm{C} 15-\mathrm{H} 16 \text { and } \mathrm{H} 21-\mathrm{C} 18-\mathrm{H} 19\end{array}$ & 1058 & & 1062 & 1045 \\
\hline$v_{48}$ & $\begin{array}{l}\tau \mathrm{HCCC}(10) \text { in } \mathrm{CH}_{3} \text { rocking }+\tau \mathrm{HCCN}(10) \text { in } \\
\mathrm{H} 33-\mathrm{C} 31-\mathrm{C} 29-\mathrm{N} 22\end{array}$ & 1038 & 1026 & 1035 & 1038 \\
\hline$v_{49}$ & $\begin{array}{l}\tau \mathrm{HCCC}(38) \text { in } \mathrm{CH}_{3} \text { rocking }+\gamma \mathrm{ONCC}(12) \text { in } \\
\mathrm{O} 24-\mathrm{C} 23-\mathrm{N} 22-\mathrm{N} 35+\delta \mathrm{HCH}(12) \text { in } \mathrm{H} 28-\mathrm{C} 25-\mathrm{H} 27\end{array}$ & 1025 & 1026 & 1019 & 1019 \\
\hline$v_{50}$ & $\begin{array}{l}\tau \mathrm{HCCN}(41) \text { in } \mathrm{H} 32-\mathrm{C} 31-\mathrm{C} 29-\mathrm{N} 22+\delta \mathrm{HCH}(13) \text { in } \\
\mathrm{H} 33-\mathrm{C} 31-\mathrm{C} 32\end{array}$ & 1025 & 1026 & 1017 & 1012 \\
\hline$v_{51}$ & $\tau \mathrm{HCCN}(20)$ in $\mathrm{H} 33-\mathrm{C} 31-\mathrm{C} 29-\mathrm{N} 22$ & 1025 & 1026 & 1012 & 1009 \\
\hline$v_{52}$ & $\tau \mathrm{HCCN}(13)$ in $\mathrm{H} 17-\mathrm{C} 15-\mathrm{C} 4-\mathrm{N} 13$ & 1025 & 1026 & 1009 & 1005 \\
\hline$v_{53}$ & $v \mathrm{CC}(33)$ in ring 1 & 979 & 990 & 998 & 999 \\
\hline$v_{54}$ & $\tau \mathrm{HCCC}(83)$ in ring 1 out of plane $\mathrm{H}$ & 970 & & 969 & 972 \\
\hline$v_{55}$ & $\begin{array}{l}\tau \mathrm{HCCC}(74) \text { in ring } 1 \text { out of plane } \mathrm{H}+\tau \mathrm{CCCC}(11) \text { in } \\
\text { ring } 1 \text { out of plane } \mathrm{C}\end{array}$ & 944 & 947 & 951 & 956 \\
\hline$v_{56}$ & $\begin{array}{l}v \mathrm{CC}(25) \text { in } \mathrm{C} 18-\mathrm{C} 15 \text { and } \mathrm{C} 25-\mathrm{C} 23+\tau \mathrm{HCCC}(10) \text { in } \\
\text { the } \mathrm{H} 20-\mathrm{C} 18-\mathrm{C} 15-\mathrm{C} 4 / \mathrm{CH}_{3} \text { rocking mode }\end{array}$ & 944 & & 948 & 955 \\
\hline$v_{57}$ & $v \mathrm{CC}(32)$ in $\mathrm{C} 18-\mathrm{C} 15$ and $\mathrm{C} 25-\mathrm{C} 23$ & 944 & & 910 & 918 \\
\hline$v_{58}$ & $v$ CC (40) in C29-C31 & 878 & 879 & 898 & 910 \\
\hline$v_{59}$ & $\begin{array}{l}\tau \mathrm{HCCC}(77) \text { in ring } 1 \text { out of ring } \mathrm{H}+\gamma \mathrm{NCCC}(10) \text { in } \\
\mathrm{N} 13-\mathrm{C} 3-\mathrm{C} 7-\mathrm{C} 2\end{array}$ & 878 & 879 & 862 & 864 \\
\hline$v_{60}$ & $\delta \mathrm{CCC}(31)$ in ring $1+v \mathrm{NC}(10)$ in $\mathrm{N} 13-\mathrm{C} 3$ & 878 & 879 & 856 & 849 \\
\hline$v_{61}$ & $\begin{array}{l}\tau \mathrm{HCCN}(42) \text { in } \mathrm{H} 17-\mathrm{C} 15-\mathrm{C} 4-\mathrm{N} 13 \text { and rocking mode } \\
\mathrm{CH}_{3} \text { in the ethyl group }+\gamma \mathrm{CNNC}(12) \text { in } \\
\mathrm{C} 1-\mathrm{N} 35-\mathrm{C} 4-\mathrm{N} 13\end{array}$ & 803 & 794 & 782 & 784 \\
\hline$v_{62}$ & $v \mathrm{NC}(11)$ in $\mathrm{C} 4-\mathrm{N} 35+v \mathrm{CC}(10)$ in $\mathrm{C} 23-\mathrm{C} 25$ & 774 & 769 & 777 & 775 \\
\hline$v_{63}$ & $\begin{array}{l}\gamma \mathrm{ONCC}(24) \text { in } \mathrm{O} 14-\mathrm{N} 35-\mathrm{C} 1-\mathrm{C} 2+\tau \mathrm{HCCC} \\
(16)+\tau \mathrm{CCCC}(12)\end{array}$ & 774 & & 774 & 767 \\
\hline$v_{64}$ & $\begin{array}{l}\tau \mathrm{HCCC}(51) \text { out of ring } \mathrm{H}+\tau \mathrm{CNCC}(10) \text { in } \\
\mathrm{C} 4-\mathrm{N} 35-\mathrm{C} 1-\mathrm{C} 2\end{array}$ & 774 & & 757 & 752 \\
\hline$v_{65}$ & $\begin{array}{l}\tau \text { CCCC (18) out of ring } 1 \mathrm{C}+\tau \mathrm{HCCC}(15) \text { out of ring } \\
1 \mathrm{H}\end{array}$ & 680 & & 682 & 677 \\
\hline$v_{66}$ & $\tau \operatorname{CCCC~}(26)$ out of ring $1 \mathrm{C}$ & 660 & 665 & 659 & 657 \\
\hline$v_{67}$ & $\delta \mathrm{CCC}(48)$ in ring 1 and $\mathrm{C} 4-\mathrm{C} 15-\mathrm{C} 18$ & 647 & 647 & 649 & 648 \\
\hline
\end{tabular}


TABle 2: Continued.

\begin{tabular}{|c|c|c|c|c|c|}
\hline \multirow{2}{*}{ Vibration number } & \multirow{2}{*}{ Assignment $(\mathrm{PED} \%)^{\mathrm{a}}$} & \multicolumn{2}{|c|}{ Observed frequencies } & \multicolumn{2}{|c|}{ Calculated frequencies in $\mathrm{cm}^{-1}$} \\
\hline & & FT-IR & Laser-Raman & B3LYP & M06-2X \\
\hline$v_{68}$ & $v \mathrm{CC}(16)$ in $\mathrm{C} 2-\mathrm{C} 3+\delta \mathrm{OCC}(15)$ in $\mathrm{O} 24-\mathrm{C} 23-\mathrm{C} 25$ & 618 & & 624 & 626 \\
\hline$v_{69}$ & $\begin{array}{l}\tau \mathrm{CNCN}(20) \text { in } \mathrm{C} 3-\mathrm{N} 13-\mathrm{C} 4-\mathrm{N} 35+\tau \mathrm{CCCC}(13) \text { in ring } \\
1+\tau \mathrm{CCCN}(13) \text { in } \mathrm{C} 3-\mathrm{C} 2-\mathrm{C} 1-\mathrm{N} 35+\gamma \mathrm{CNNC}(12) \text { in } \\
\mathrm{C} 1-\mathrm{N} 35-\mathrm{N} 22-\mathrm{C} 23\end{array}$ & 601 & 598 & 608 & 605 \\
\hline$v_{70}$ & $\delta \mathrm{OCC}(27)$ in $\mathrm{O} 24-\mathrm{C} 23-\mathrm{C} 25+v \mathrm{CC}(11)$ in $\mathrm{C} 15-\mathrm{C} 4$ & 601 & 598 & 591 & 592 \\
\hline$v_{71}$ & $\gamma \mathrm{ONCC}(39)$ in $\mathrm{O} 24-\mathrm{N} 22-\mathrm{C} 29-\mathrm{C} 25$ & 572 & 580 & 584 & 582 \\
\hline$v_{72}$ & $\begin{array}{l}\gamma \mathrm{ONCC}(15) \text { in } \mathrm{O} 24-\mathrm{N} 22-\mathrm{C} 29-\mathrm{C} 25+\delta \mathrm{OCC}(15) \text { in } \\
\mathrm{O} 24-\mathrm{C} 23-\mathrm{C} 25\end{array}$ & 562 & 542 & 557 & 556 \\
\hline$v_{73}$ & $\gamma \mathrm{ONCC}(50)$ in $\mathrm{O} 24-\mathrm{N} 22-\mathrm{C} 29-\mathrm{C} 25$ & 543 & 542 & 551 & 548 \\
\hline$v_{74}$ & $\delta \mathrm{CCC}(14)$ in ring 1 & 520 & & 527 & 526 \\
\hline$v_{75}$ & $\begin{array}{l}\tau \mathrm{CCCC}(18) \text { out of ring } 1+\gamma \mathrm{NCCC}(12) \text { in } \\
\mathrm{N} 13-\mathrm{C} 3-\mathrm{C} 2-\mathrm{C} 6+\gamma \mathrm{CNNC}(10) \text { in } \mathrm{C} 4-\mathrm{N} 3-\mathrm{N} 22-\mathrm{C} 23\end{array}$ & 520 & & 507 & 503 \\
\hline$v_{76}$ & $\delta \mathrm{CCC}(27)$ in $\mathrm{C} 4-\mathrm{C} 15-\mathrm{C} 18$ and in ring 1 & 461 & 463 & 452 & 449 \\
\hline$v_{77}$ & $\begin{array}{l}\tau \text { CCCC }(24) \text { out of ring } 1+\gamma \text { NCCC }(10) \text { in } \\
\text { N13-C3-C2-C6 }\end{array}$ & 461 & 420 & 446 & 444 \\
\hline$v_{78}$ & $\tau$ CCCC $(27)$ out of ring 1 & 419 & & 407 & 409 \\
\hline$v_{79}$ & $\begin{array}{l}\delta \mathrm{NNC}(15) \text { in } \mathrm{N} 22-\mathrm{N} 35-\mathrm{C} 4+\delta \mathrm{OCC}(13) \text { in } \\
\mathrm{O} 14-\mathrm{C} 1-\mathrm{C} 2+v \mathrm{NC}(11) \text { in } \mathrm{N} 22-\mathrm{C} 23\end{array}$ & & & 394 & 400 \\
\hline$v_{80}$ & $\begin{array}{l}\delta \mathrm{OCC}(14) \text { in } \mathrm{O} 14-\mathrm{C} 1-\mathrm{C} 2+\delta \mathrm{CCN}(18) \text { in } \\
\mathrm{C} 25-\mathrm{C} 23-\mathrm{N} 22\end{array}$ & & & 391 & 393 \\
\hline$v_{81}$ & $\delta \mathrm{CCN}(57)$ in $\mathrm{C} 25-\mathrm{C} 23-\mathrm{N} 22$ & & 365 & 355 & 363 \\
\hline$v_{82}$ & $\begin{array}{l}\delta \mathrm{CNN}(25) \text { in } \mathrm{C} 29-\mathrm{N} 22-\mathrm{N} 35+\delta \mathrm{CCC}(12) \text { in } \\
\mathrm{C} 18-\mathrm{C} 15-\mathrm{C} 4\end{array}$ & & 365 & 346 & 353 \\
\hline$v_{83}$ & $\begin{array}{l}\delta \mathrm{CCN}(18) \text { in } \mathrm{C} 25-\mathrm{C} 23-\mathrm{N} 22+\delta \mathrm{CCC}(11) \text { in } \\
\mathrm{C} 18-\mathrm{C} 15-\mathrm{C} 4\end{array}$ & & 328 & 314 & 325 \\
\hline$v_{84}$ & $\begin{array}{l}\tau \mathrm{CCCC}(30) \text { out of ring } 1+\gamma \mathrm{CNNC}(12) \text { in } \\
\mathrm{C} 3-\mathrm{C} 4-\mathrm{N} 35-\mathrm{C} 1\end{array}$ & & 297 & 291 & 289 \\
\hline$v_{85}$ & $\delta \mathrm{NCC}(36)$ in $\mathrm{N} 22-\mathrm{C} 23-\mathrm{C} 25$ & & 297 & 287 & 285 \\
\hline$v_{86}$ & $\tau \mathrm{HCCN}(56)$ & & 218 & 229 & 251 \\
\hline$v_{87}$ & $\begin{array}{l}\delta \mathrm{CNC}(25) \text { in } \mathrm{C} 23-\mathrm{N} 22-\mathrm{C} 29+\delta \mathrm{CCN}(13) \text { in } \\
\mathrm{C} 15-\mathrm{C} 4-\mathrm{N} 13+\tau \mathrm{HCCC}(10)\end{array}$ & & 218 & 202 & 226 \\
\hline$v_{88}$ & $\tau \mathrm{HCCC}(45)$ & & 218 & 194 & 211 \\
\hline$v_{89}$ & $\tau \mathrm{HCCN}(83)$ in $\mathrm{H} 33-\mathrm{C} 31-\mathrm{C} 29-\mathrm{N} 22$ & & 162 & 182 & 203 \\
\hline$v_{90}$ & $\begin{array}{l}\tau \mathrm{CNCC}(37) \text { in } \mathrm{C} 4-\mathrm{N} 13-\mathrm{C} 3-\mathrm{C} 2+\gamma \mathrm{NCCC}(15) \text { in } \\
\mathrm{N} 13-\mathrm{C} 4-\mathrm{C} 15-\mathrm{C} 18\end{array}$ & & 162 & 156 & 156 \\
\hline$v_{91}$ & $\begin{array}{l}\delta \mathrm{CCN}(21) \text { in } \mathrm{C} 15-\mathrm{C} 4-\mathrm{N} 13+\delta \mathrm{CCC}(18) \text { in } \\
\mathrm{C} 18-\mathrm{C} 15-\mathrm{C} 4+\delta \mathrm{CNC}(10) \text { in } \mathrm{C} 1-\mathrm{N} 35-\mathrm{C} 4\end{array}$ & & 132 & 153 & 153 \\
\hline$v_{92}$ & $\begin{array}{l}\tau \mathrm{CNCN}(35) \text { in } \mathrm{C} 3-\mathrm{N} 13-\mathrm{C} 4-\mathrm{N} 35+\gamma \mathrm{NCCN}(12) \text { in } \\
\mathrm{N} 13-\mathrm{C} 4-\mathrm{C} 15-\mathrm{N} 35\end{array}$ & & 132 & 122 & 120 \\
\hline$v_{93}$ & $\begin{array}{l}\tau \mathrm{CNCN}(25) \text { in } \mathrm{C} 3-\mathrm{N} 13-\mathrm{C} 4-\mathrm{N} 35+\gamma \mathrm{CNNC} \\
(16)+\gamma \mathrm{NCCC}(10)+\tau \mathrm{CCCC}(10)\end{array}$ & & & 105 & 97 \\
\hline$v_{94}$ & $\gamma \mathrm{CNCN}(52)+\gamma \mathrm{NCCN}(12)+\tau \mathrm{CNCN}(10)$ & & & 95 & 88 \\
\hline$v_{95}$ & $\tau \mathrm{CCNN}(32)+\tau \mathrm{CNCN}(28)+\gamma \mathrm{CNCN}(10)$ & & & 71 & 71 \\
\hline$v_{96}$ & $\tau \mathrm{CCNN}(71)+\delta \mathrm{NNC}(14)$ & & & 63 & 62 \\
\hline$v_{97}$ & $\tau \mathrm{CCNN}(61)+\gamma \mathrm{NCCN}(15)$ & & & 60 & 57 \\
\hline$v_{98}$ & $\tau \mathrm{CCNN}(37)+\gamma \mathrm{NCCN}(13)+\tau \mathrm{CNCN}$ & & & 43 & 44 \\
\hline$v_{99}$ & $\tau \mathrm{CNCN}(45)+\gamma \mathrm{NCCN}(29)$ & & & 39 & 37 \\
\hline
\end{tabular}


TABLe 2: Continued.

\begin{tabular}{|c|c|c|c|c|c|}
\hline \multirow{2}{*}{ Vibration number } & \multirow{2}{*}{ Assignment (PED\%) ${ }^{\mathrm{a}}$} & \multicolumn{2}{|c|}{ Observed frequencies } & \multicolumn{2}{|c|}{ Calculated frequencies in $\mathrm{cm}^{-1}$} \\
\hline & & FT-IR & Laser-Raman & B3LYP & M06-2X \\
\hline$R^{2}$ & & & & 0.9998 & 0.9997 \\
\hline
\end{tabular}

$v$, stretching; $\delta$, in-plane bending; $\gamma$, out-of-plane bending; $\tau$, torsion.

${ }^{a}$ Potential energy distribution (PED); values less than $10 \%$ are not shown.

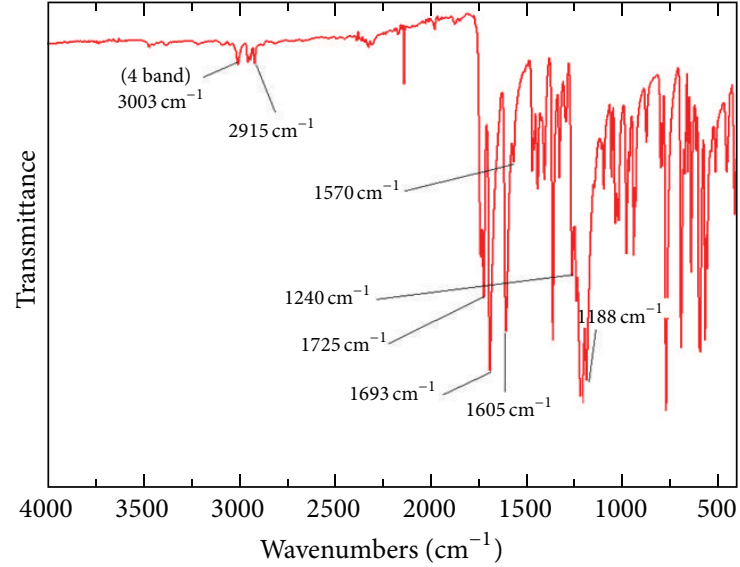

— Experimental IR

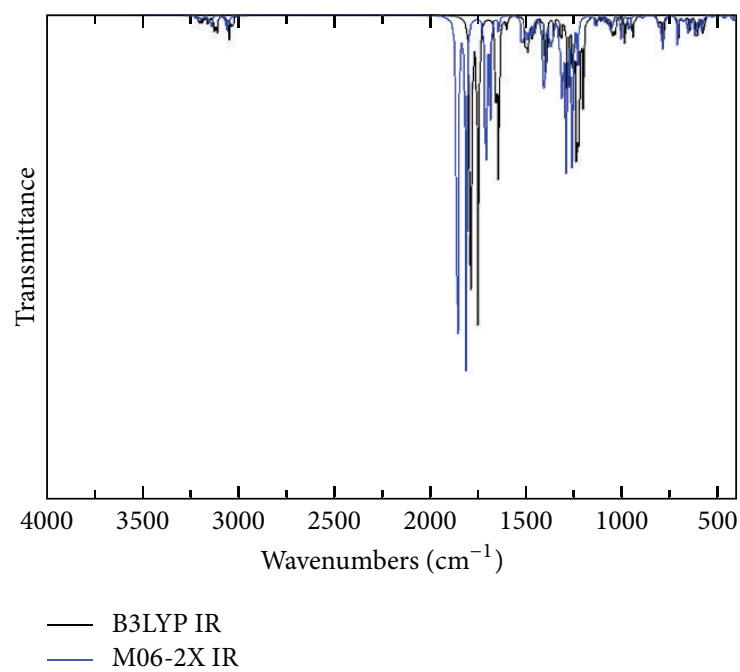

Figure 2: Comparison of observed and calculated infrared spectra of compound 2 .

detailed potential energy distribution (PED) assignments are summarized in Table 2 . The harmonic frequencies calculated for the title molecule relate to the gaseous phase, but the experimental ones are obtained for the solid phase. As a result, disagreement between the calculated frequencies and some of the experimental (observed) frequencies is to be expected. A PED analysis was carried out in order to introduce detailed vibrational assignments for compound 2. Within each fundamental wave number, the calculated modes are numbered downwards from the largest to the smallest frequency.

The harmony between the experimental and calculated wavenumbers is shown in Figure 4, which indicates that the

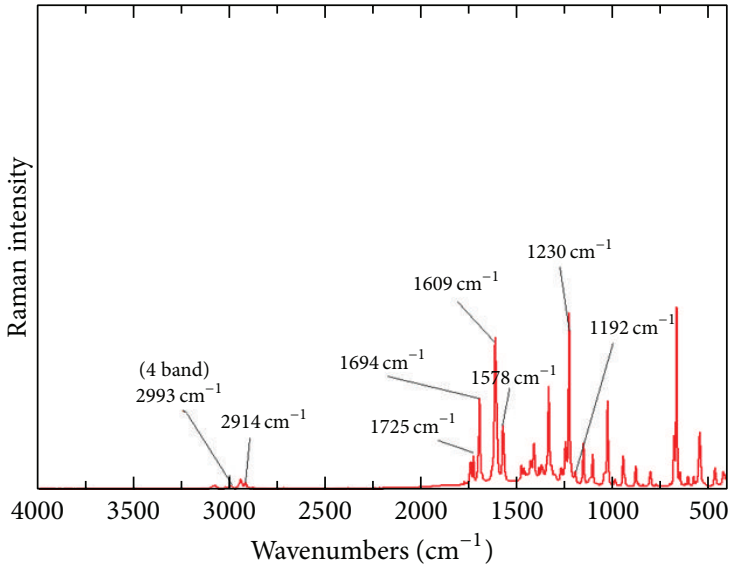

_ Experimental Ra

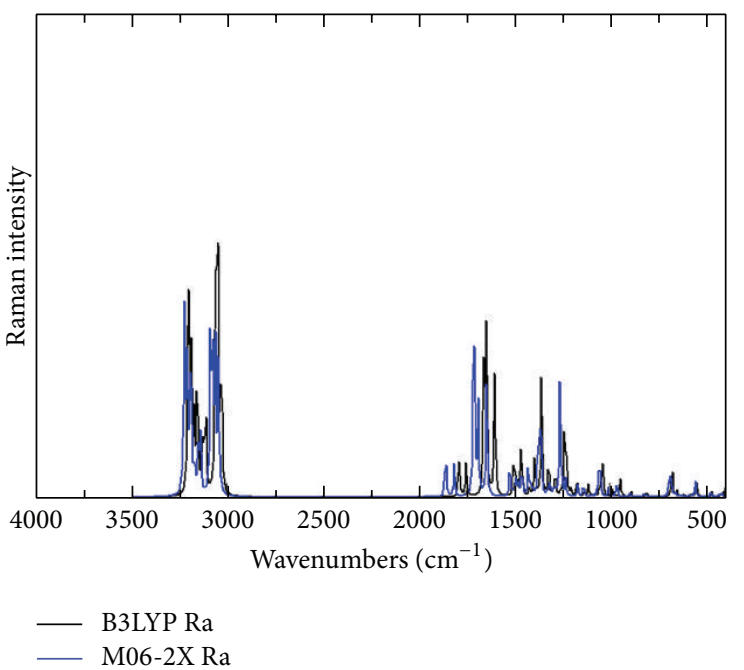

FIGURE 3: Comparison of observed and calculated Raman spectra of compound 2 .

experimental frequencies correlate well with the calculated ones, particularly for B3LYP. The relations between the experimental and calculated wavenumbers are linear and given by the following:

$$
\begin{array}{ll}
v_{\mathrm{Cal}}=0.9986 v_{\exp }-0.0726 & \text { for B3LYP method, } \\
v_{\mathrm{Cal}}=0.9927 v_{\exp }+5.7907 & \text { for M06-2X method. }
\end{array}
$$

The correlation coefficients $\left(R^{2}\right.$ values) between the experimental and calculated wavenumbers were calculated as $R^{2}=0.9998$ for B3LYP and $R^{2}=0.9997$ for M06$2 X$. This indicates that the calculation methods gave reasonable agreement with the experimental values, particularly 

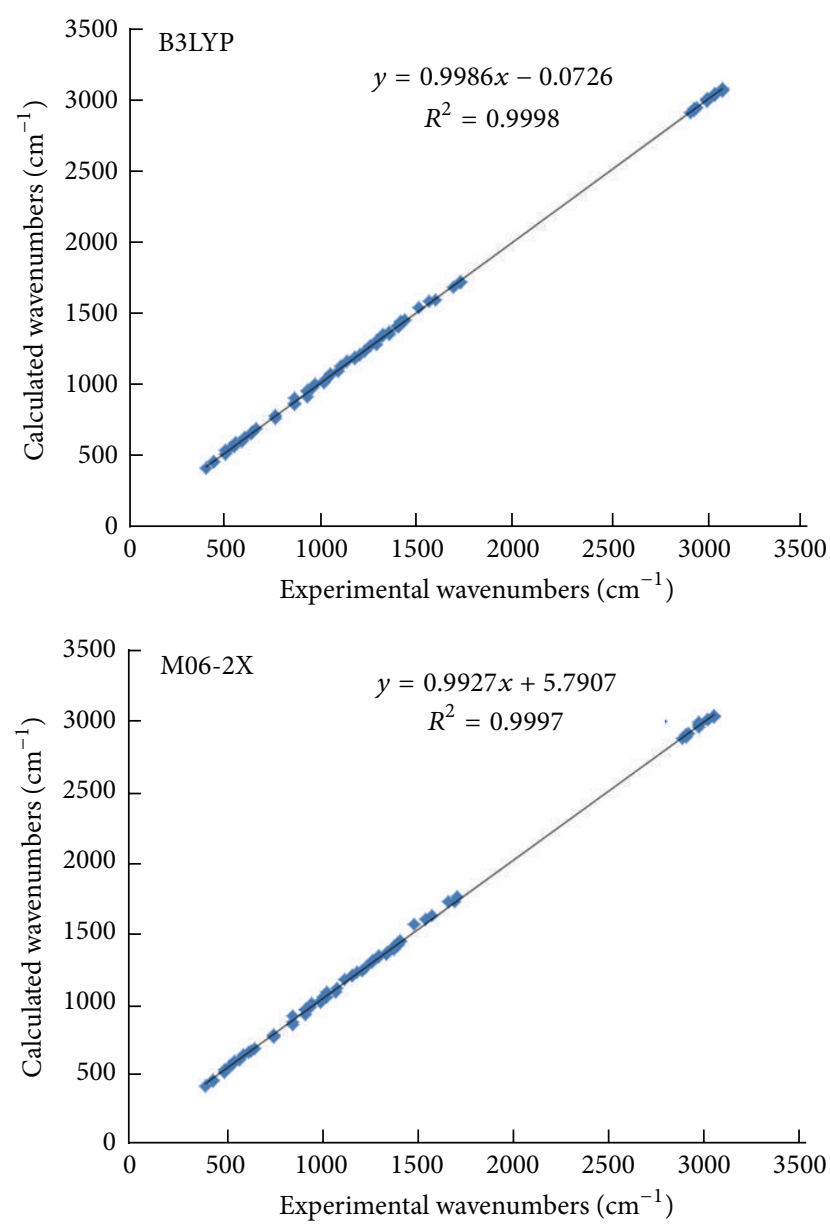

FIgURE 4: Correlation graphics of experimental and theoretical (scaled) wavenumbers of 2 .

in the case of the B3LYP method. However, this masks significant differences between experimental and calculated values for a few of the vibrational frequencies, which are worthy of individual mention.

4.3.1. Quinazolinone Carbonyl Group Vibrations. The carbonyl group has a strong $\mathrm{C}=\mathrm{O}$ stretching vibration around 1850 $1550 \mathrm{~cm}^{-1}$ [45]. Differences of $20-30 \mathrm{~cm}^{-1}$ are used to help recognize particular functional groups such as ester, ketone, or amide groups. For compound 2, the quinazolinone $\nu \mathrm{C}=\mathrm{O}$ mode is observed as a strong band at $1693 \mathrm{~cm}^{-1}$ in the IR spectrum and at $1694 \mathrm{~cm}^{-1}$ in the Laser-Raman spectrum. The calculated values are $1683 \mathrm{~cm}^{-1}$ (B3LYP) and $1721 \mathrm{~cm}^{-1}$ (M06-2X). This demonstrates that although the correlation coefficients $R^{2}$ are high, individual calculated frequencies can differ significantly from each other and from the experimental values, and in the case of the M06-2X method the calculated carbonyl stretching frequency differs from the experimental value by a sufficiently large margin that it could not be relied on to identify the functional group. In other cases, however, the level of agreement may be greater. For example, $\gamma \mathrm{ONCC}(\mathrm{O} 14-\mathrm{N} 35-\mathrm{C} 1-\mathrm{C} 2)$ is seen at $774 \mathrm{~cm}^{-1}$ in the IR spectrum and calculated at $774 \mathrm{~cm}^{-1}$ by B3LYP and $767 \mathrm{~cm}^{-1}$ by M06-2X. Bending modes $\delta$ OCC (O14-C1-C2) were not seen in either the IR spectrum or the Raman spectrum, but two such modes were calculated to be at 394 (B3LYP)/400 (M06-2X) and 391 (B3LYP)/393 (M06-2X) $\mathrm{cm}^{-1}$. For similar quinazoline derivatives $[46,47]$ the $\nu \mathrm{C}=\mathrm{O}$ mode was reported at 1652 (3-\{[(2-hydroxyphenyl) methylene]amino\}-2-phenylquinazolin-4(3H)-one) and $1673 \mathrm{~cm}^{-1}$ (3-\{[(4-fluorophenyl) methylene]amino\}-2-phenylquinazolin$4(3 \mathrm{H})$-one) in the IR spectrum and at 1656 and $1661 \mathrm{~cm}^{-1}$, respectively, in the Raman spectrum. For 3-(4-methoxybenzylideneamino)-2-phenyl-3H-quinazolin-4-one this mode was seen as a strong band at $1683 \mathrm{~cm}^{-1}$ in the IR spectrum and at $1682 \mathrm{~cm}^{-1}$ in the Raman spectrum and calculated to be $1690 \mathrm{~cm}^{-1}$ using the B3LYP/6-31G ${ }^{*}$ basis set [42].

4.3.2. C15H16, 17 and C18H19, 20, 21 Group Vibrations. The $\mathrm{CH}_{2}$ group has 6 vibrational modes (one asymmetrical and one symmetrical stretching, one scissoring, one wagging, one twisting and rocking). The asymmetric stretch $v_{\mathrm{as}} \mathrm{CH}_{2}$, the symmetric stretch $v_{\mathrm{s}} \mathrm{CH}_{2}$, the scissoring vibrations $\delta \mathrm{CH}_{2}$, and the wagging vibration $\omega \mathrm{CH}_{2}$ appear at $3000 \pm 20$, $2900 \pm 25,1440 \pm 10$, and $1340 \pm 25 \mathrm{~cm}^{-1}$, respectively [48, 49]. For compound 2, agreement between the experimental values and those calculated by B3LYP was generally good but there was less good agreement with those calculated by M06-2X (see Table 2). For example, the $\mathrm{CH}_{2}$ asymmetrical stretching mode was seen at $2934 \mathrm{~cm}^{-1}$ in the IR spectrum and $2938 \mathrm{~cm}^{-1}$ in the Raman spectrum. This asymmetrical stretching mode was calculated to be at 2939 (B3LYP)/2922 (M06-2X) $\mathrm{cm}^{-1}$. The $\mathrm{CH}_{2}$ symmetrical stretching mode was observed at $2915 \mathrm{~cm}^{-1}$ in the IR spectrum and $2914 \mathrm{~cm}^{-1}$ in the Raman spectrum. The symmetrical stretching mode was calculated to be at $2911 \mathrm{~cm}^{-1}$ by B3LYP, but $2888 \mathrm{~cm}^{-1}$ by M06-2X. Similarly, the $\mathrm{CH}_{2}$ scissoring and wagging modes were seen at 1409 and $1328 \mathrm{~cm}^{-1}$ in the IR spectrum, and 1406 and $1333 \mathrm{~cm}^{-1}$ in the Laser-Raman spectrum, respectively. These modes were calculated to be at 1406 (B3LYP)/1389 (M06-2X) and 1337 (B3LYP)/1331 (M06-2X) $\mathrm{cm}^{-1}$, respectively. $\mathrm{CH}_{2}$ twisting and rocking modes appeared at $1260 \pm$ 10 and $800 \pm 25 \mathrm{~cm}^{-1}$, respectively [49]. For compound 2, the $\mathrm{CH}_{2}$ twisting and rocking modes were seen at 1240 and $803 \mathrm{~cm}^{-1}$ in the IR spectrum, 1247 and $794 \mathrm{~cm}^{-1}$ in the LaserRaman spectrum, respectively, and calculated to be at 1246 (B3LYP)/1246 (M06-2X) and 782 (B3LYP)/784 (M06-2X) $\mathrm{cm}^{-1}$, respectively.

The $\mathrm{CH}_{3}$ group has nine vibrational modes (two asymmetrical and one symmetrical stretching modes, two antisymmetrical deformations, one symmetrical deformation, two rocking modes, and 1 twisting mode). The $\mathrm{CH}_{3}$ asymmetric stretching vibrations are expected in the $2950-3050 \mathrm{~cm}^{-1}$ region and $\mathrm{CH}_{3}$ symmetric vibrations in the $2900-2950 \mathrm{~cm}^{-1}$ region [48, 49]. For compound 2 the asymmetric stretching modes were calculated to be at 3003 (B3LYP)/2988 (M06-2X) and 2989 (B3LYP)/2977 (M06-2X) $\mathrm{cm}^{-1}$, and the symmetric mode at 2928 (B3LYP)/2904 (M06-2X) $\mathrm{cm}^{-1}$. Asymmetric stretching modes were observed at $3003 \mathrm{~cm}^{-1}$ in the IR spectrum and at $2993 \mathrm{~cm}^{-1}$ in the Laser-Raman spectrum (the two 
bands appearing within a single envelope in each case), while symmetric stretching modes were observed at $2934 \mathrm{~cm}^{-1}$ in the IR and $2914 \mathrm{~cm}^{-1}$ in the Laser-Raman spectrum. Again, therefore, the B3LYP method is a better predictor of the experimental values than the M06-2X method. The same trend is observed for some of the other vibrational modes. For example, the symmetrical bending deformation, $\delta_{\mathrm{s}} \mathrm{CH}_{3}$, is expected at $1380 \pm 25 \mathrm{~cm}^{-1}$ [48]. The calculated value was 1366 (B3LYP)/1353 (M06-2X) $\mathrm{cm}^{-1}$, while the observed value was $1364 \mathrm{~cm}^{-1}$ in the IR spectrum and $1363 \mathrm{~cm}^{-1}$ in the LaserRaman spectrum. Similarly, aromatic molecules display a methyl rocking in the neighborhood of $1045 \mathrm{~cm}^{-1}$, and a second rocking in the region of $970 \pm 70 \mathrm{~cm}^{-1}$, which is more difficult to find among the $\mathrm{C}-\mathrm{H}$ out-of-plane deformations [48]. For compound 2, these $\rho \mathrm{CH}_{3}$ modes were observed at 1038 and $944 \mathrm{~cm}^{-1}$ in the IR spectrum; the first of these bands was observed at $1026 \mathrm{~cm}^{-1}$ in the Laser-Raman spectrum, but the second rocking mode was not observed. These bands were calculated at 1035 (B3LYP)/1038 (M06-2X) and 948 (B3LYP)/955 (M06-2X) $\mathrm{cm}^{-1}$, respectively.

Although the M06-2X calculated frequencies of some vibrational modes were a little closer to the observed values than the ones calculated by B3LYP, in the overwhelming majority of cases B3LYP provided calculated values that were closer to the observed values (see Table 2). This perspective is more easily seen in the graphical experimental and calculated spectra shown in Figure 2 (IR) and Figure 3 (Raman). Figure 3 also reveals that the calculated intensities of some of the bands in the Raman spectra were substantially different from the experimentally observed intensities.

4.3.3. Diacetylamino Group Vibrations. For compound 2, $\nu \mathrm{C}=\mathrm{O}(\mathrm{C} 23-\mathrm{O} 24$ and $\mathrm{C} 29-\mathrm{O} 30)$ stretching modes were observed at 1735 (IR)/1743 (Raman) and 1725 (IR)/1725 (Raman) $\mathrm{cm}^{-1}$, and calculated at 1720 (B3LYP)/1764 (M062X) and 1718 (B3LYP)/1721 (M06-2X) $\mathrm{cm}^{-1}$. For 3-nitroacetanilide this mode was observed at $1676 \mathrm{~cm}^{-1}$ in the IR spectrum and calculated at $1774 \mathrm{~cm}^{-1}$ by using the $\mathrm{HF} / 6-31 \mathrm{G}^{*}$ method [50]. For compound 2, two C-N stretching modes (N22-C23 and N22-C29) were observed at 1240 (IR)/1230 (Raman) and 1188 (IR)/1192 (Raman) $\mathrm{cm}^{-1}$, respectively, but the latter band contained two $\mathrm{C}-\mathrm{N}$ stretching frequencies within the envelope; all three frequencies were calculated, at 1235 (B3LYP)/1227 (M06-2X), 1190 (B3LYP)/1198 (M06-2X), and 1180 (B3LYP)/1195 (M06-2X) $\mathrm{cm}^{-1}$.

In the acetyl group, the two $\mathrm{CH}_{3}$ groups have 18 vibrational modes (4 asymmetrical and 2 symmetrical stretching modes, 4 antisymmetrical and 2 symmetrical deformations, 4 rocking modes, and 2 twisting modes). The $\mathrm{CH}_{3}$ asymmetric stretching vibrations are expected to be at $2950-3050 \mathrm{~cm}^{-1}$ and the $\mathrm{CH}_{3}$ symmetric vibrations to be at $2900-2950 \mathrm{~cm}^{-1}$ $[48,49]$. For compound 2 , the symmetric stretching modes were seen at 2949 and $2934 \mathrm{~cm}^{-1}$ in the IR spectrum and at $2938 \mathrm{~cm}^{-1}$ in the Laser-Raman spectrum. They were calculated to be at 2943 (B3LYP)/2929 (M06-2X) and 2934 (B3LYP)/2916 (M06-2X) $\mathrm{cm}^{-1}$, again showing that the B3LYP method gives better agreement with experimental values.
4.3.4. Quinazolinone Group Vibrations. In quinazoline compounds, $\mathrm{C}=\mathrm{N}$ stretching bands are generally observed at $1627-1566 \mathrm{~cm}^{-1}$ [51-53], while for conjugated azines the $\nu \mathrm{C}=\mathrm{N}$ mode has been reported to appear around $1553 \mathrm{~cm}^{-1}$ [54]. For compound 2, the $(\mathrm{C} 4=\mathrm{N} 13)$ bands were seen at 1605 (IR)/1609 (Raman) and 1570 (IR)/1578 (Raman) $\mathrm{cm}^{-1}$ and calculated to be at 1593 (B3LYP)/1623 (M06-2X) and 1580 (B3LYP)/1601 (M06-2X) $\mathrm{cm}^{-1}$. Again, the B3LYP method provides the closer fit with the experimental values. Other C-N stretching bands [55] generally occur around 1100$1300 \mathrm{~cm}^{-1}$, while aromatic $\mathrm{C}-\mathrm{H}$ stretching typically exhibits several weak to moderate bands above $3000 \mathrm{~cm}^{-1}$ [56]. Aromatic $\mathrm{C}-\mathrm{H}$ in-plane bending vibrations usually occur in the region of $1390-990 \mathrm{~cm}^{-1}$ and are very useful for characterization purposes [57], while $\mathrm{C}-\mathrm{H}$ out-of-plane deformations generally occur around 1000 to $700 \mathrm{~cm}^{-1}$, with bands having higher wavenumbers being weaker than those absorbing at lower wavenumbers [58]. Table 2 shows the experimental and calculated values for compound 2 .

Ring $\mathrm{C}=\mathrm{C}$ stretching vibrations usually occur around $1625-1430 \mathrm{~cm}^{-1}$ [59]. For benzenoid compounds there are two or three bands due to skeletal vibrations; the strongest band is at $c a .1500 \mathrm{~cm}^{-1}$. The observed and calculated values for compound 2 are shown in Table 2.

4.4. HOMO-LUMO Analysis. Chemical stability is mainly influenced by the frontier orbitals (HOMO and LUMO). The HOMO represents electron-donating capability, while the LUMO represents electron accepting capability [60]. The HOMO and LUMO energies of 2 were calculated by the B3LYP/6-311++G(d,p) (Figure 5; positive phase represented in red and negative phase represented in green) and M062X/6-311++G(d,p) methods. A large HOMO-LUMO gap implies a chemically "hard" molecule and a small HOMOLUMO gap implies a "soft" molecule. The chemical reactivity of a molecule is also related to its "hardness," molecules with lower HOMO-LUMO gaps being more reactive [61]. The frontier molecular orbital energy gap therefore helps in understanding the kinetic stability and reactivity of molecules $[62,63]$.

The HOMO-LUMO energy gap calculated for compound 2 is $5.119590424 \mathrm{e.V}$ by B3LYP/6-311++G(d,p) and 7.459788024 e.V by M06-2X/6-311++G(d,p). This energy gap reflects the chemical activity of the molecule and influences its biological activity. As is evident from Figure 5, the HOMO is located on the quinazoline rings, the acetylamino group, and over the $\mathrm{Cl}=\mathrm{O} 14$ carbonyl group. The LUMO is more focused on the quinazoline ring and the $\mathrm{Cl}=\mathrm{O} 14$ carbonyl group and partially over the C29 atom.

The ionization energy $(I)$ and electron affinity $(A)$ can be expressed by HOMO and LUMO orbital energies as $I=$ $-E_{\mathrm{HOMO}}$ and $A=-E_{\mathrm{LUMO}}$. The global hardness $\eta=$ $1 / 2\left(E_{\mathrm{LUMO}}-E_{\mathrm{HOMO}}\right)$. The ionization energy, along with electron affinity, can be used to give electronic chemical potential, $\mu=1 / 2\left(E_{\mathrm{LUMO}}+E_{\mathrm{HOMO}}\right)$, the global electrophilicity index $\psi=\mu^{2} / 2 \eta$, and the softness $\zeta=1 / \eta$ [64]. Such parameters were calculated and are tabulated in Table 3 . The ionization 
TABLE 3: Comparison of HOMO-LUMO energy gaps and related molecular properties of 2.

\begin{tabular}{lcc}
\hline Molecular properties & B3LYP/6-311++G(d,p) & M06-2X/6-311++G(d,p) \\
\hline Energies (a.u) & -932.460118 & -932.085968 \\
$E_{\text {HOMO }}(\mathrm{e} . \mathrm{V})$ & -6.938958 & -8.084022128 \\
$E_{\mathrm{LUMO}}(\mathrm{e} . \mathrm{V})$ & -1.819367576 & -0.828865336 \\
Energy gap $(\mathrm{e} . \mathrm{V})$ & 5.119590424 & 7.459788024 \\
Ionization potential $(I)$ & 6.938958 & 8.084022128 \\
Electron affinity $(A)$ & 1.819367576 & 0.828865336 \\
Global hardness $(\eta)$ & 2.5597995212 & 3.627677972 \\
Chemical potential $(\mu)$ & 4.37912788 & 4.4564433732 \\
Electrophilicity $(\psi)$ & 3.74581419 & 2.73727385 \\
Softness $(\zeta)$ & 0.390655593 & 0.2756584261 \\
Dipole moment $($ debye $)$ & 2.9159 & 2.8371 \\
\hline
\end{tabular}

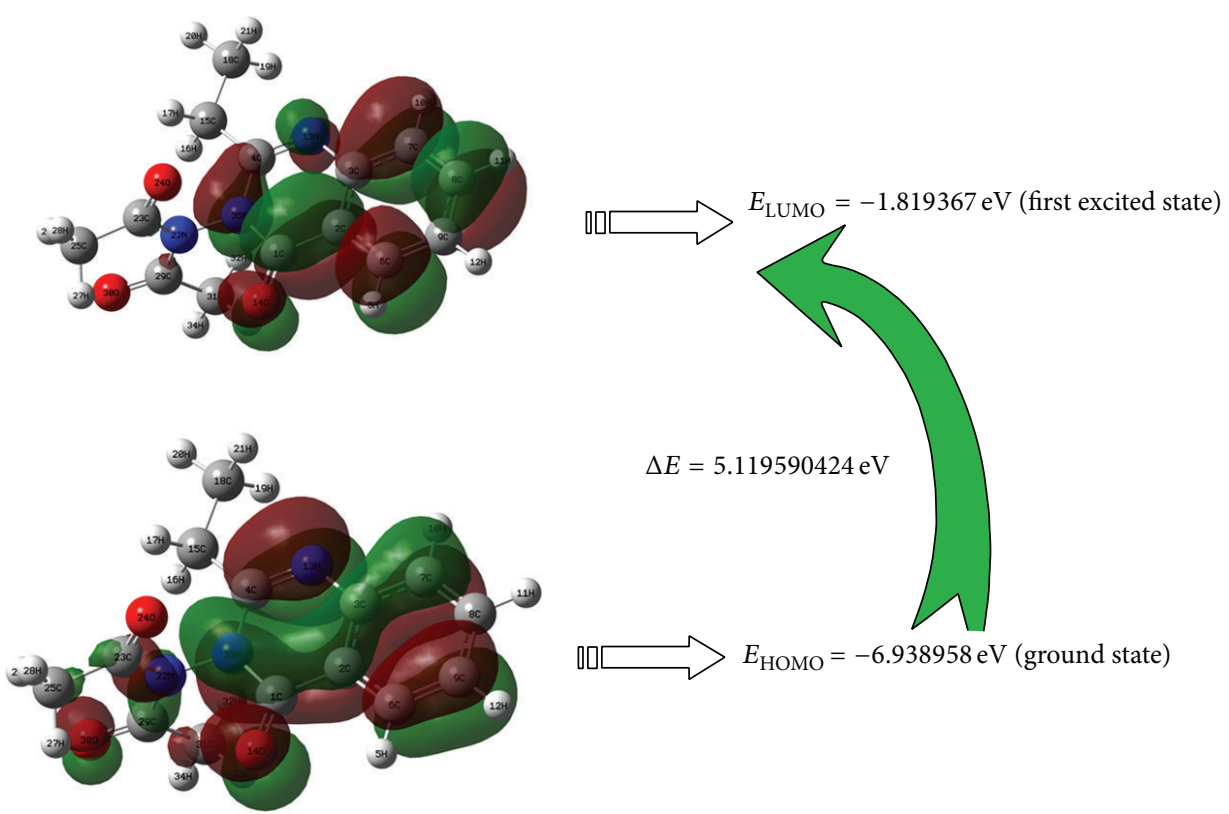

Figure 5: Calculated HOMO-LUMO plots of title compound 2.

potentials given by the B3LYP and M06-2X methods for 2 are $6.938958 \mathrm{eV}$ and $8.084022128 \mathrm{eV}$, respectively. The HOMO and LUMO energies of 2-quinazolinone and 4quinazolinone calculated by B3LYP/6-311G(d), 6-311+G(d,p), and $6-311++G(d, p)$ sets have been reported previously [58]. For both molecules, LUMOs are distributed uniformly over the entire molecules and show antibonding character. For both molecules, the HOMOs are shifted mainly towards the pyrimidinone and some part of the benzene ring [58].

\section{Conclusion}

In this work, the vibrational modes of newly synthesized 3(diacetylamino)-2-ethyl-3H-quinazolin-4-one (2) were studied experimentally by use of FT-IR and Laser-Raman spectra and computationally using DFT/B3LYP and M06-2X methods. The vibrational harmonic frequencies, optimized geometric parameters, molecular orbital energies, and other properties related to HOMO and LUMO energy values of compound 2 were calculated using DFT/B3LYP and M06-2X methods with the $6-311++G(d, p)$ basis set. The assignments of the vibrational frequencies were made with the help of potential energy distribution (PED) analysis using VEDA 4 software. The theoretical optimized geometric parameters and vibrational frequencies have been found to be in good agreement with the corresponding experimental data and results in the literature. The calculated HOMO and LUMO orbitals and their energies have been used to gain understanding of charge transfer within compound 2 .

\section{Conflict of Interests}

The authors declare that there is no conflict of interests regarding the publication of this paper. 


\section{Acknowledgment}

The authors would like to extend their appreciation to the Deanship of Scientific Research at King Saud University for funding this research through the research group project RGP-VPP-239.

\section{References}

[1] V. Alagarsamy, V. R. Solomon, and K. Dhanabal, "Synthesis and pharmacological evaluation of some 3-phenyl-2-substituted$3 \mathrm{H}$-quinazolin-4-one as analgesic, anti-inflammatory agents," Bioorganic and Medicinal Chemistry, vol. 15, no. 1, pp. 235-241, 2007.

[2] R. Rohini, P. M. Reddy, K. Shanker, A. Hu, and V. Ravinder, "Antimicrobial study of newly synthesized 6-substituted indolo[1,2c]quinazolines," European Journal of Medicinal Chemistry, vol. 45, no. 3, pp. 1200-1205, 2010.

[3] V. Gupta, S. K. Kashaw, V. Jatav, and P. Mishra, "Synthesis and antimicrobial activity of some new 3-[5-(4-substituted) phenyl1,3,4-oxadiazole-2yl]-2- styrylquinazoline-4(3H)-ones," Medicinal Chemistry Research, vol. 17, no. 2, pp. 205-211, 2008.

[4] V. Chandregowda, A. K. Kush, and G. C. Reddy, "Synthesis and in vitro antitumor activities of novel 4-anilinoquinazoline derivatives," European Journal of Medicinal Chemistry, vol. 44, no. 7, pp. 3046-3055, 2009.

[5] M. F. Abdel-Megeed, B. E. Badr, M. M. Azaam, and G. A. El-Hiti, "Synthesis, antimicrobial and anticancer activities of a novel series of diphenyl 1-(pyridin-3-yl)ethylphosphonates," Bioorganic and Medicinal Chemistry, vol. 20, no. 7, pp. 22522258, 2012.

[6] S. Sasmal, G. Balaji, H. R. K. Reddy et al., "Design and optimization of quinazoline derivatives as melanin concentrating hormone receptor 1 (MCHR1) antagonists," Bioorganic \& Medicinal Chemistry Letters, vol. 22, no. 9, pp. 3157-3162, 2012.

[7] M. S. Malamas and J. Millen, "Quinazolineacetic acids and related analogues as aldose reductase inhibitors," Journal of Medicinal Chemistry, vol. 34, no. 4, pp. 1492-1503, 1991.

[8] J. Ma, B. Han, J. Song et al., "Efficient synthesis of quinazoline2,4(1H,3H)-diones from $\mathrm{CO}_{2}$ and 2-aminobenzonitriles in water without any catalyst," Green Chemistry, vol. 15, no. 6, pp. 1485-1489, 2013.

[9] L. Xu, Y. Jiang, and D. Ma, "Synthesis of 3-substituted and 2,3disubstituted quinazolinones via $\mathrm{Cu}$-catalyzed aryl amidation," Organic Letters, vol. 14, no. 4, pp. 1150-1153, 2012.

[10] M. Adib, E. Sheikhi, and H. R. Bijanzadeh, "One-pot threecomponent synthesis of 4(3H)-quinazolinones from benzyl halides, isatoic anhydride, and primary amines," Synlett, vol. 23, no. 1, Article ID D13011ST, pp. 85-88, 2012.

[11] K. Smith, G. A. El-Hiti, and M. F. Abdel-Megeed, "Regioselective lithiation of chiral 3-acylamino-2-alkylquinazolin-4(3H)ones: application in synthesis," Synthesis, no. 13, pp. 2121-2130, 2004.

[12] G. A. El-Hiti, "A simple procedure for the side-chain substitution of 2-alkyl-3H-quinazoline-4-thiones: application in synthesis," Synthesis, no. 3, pp. 363-368, 2004.

[13] G. A. El-Hiti, "Application of organolithium in organic synthesis: a simple and convenient procedure for the synthesis of more complex 6-substituted 3H-quinazolin-4-ones," Monatshefte fur Chemie, vol. 135, no. 3, pp. 323-331, 2004.
[14] G. A. El-Hiti, A. S. Hegazy, M. H. Alotaibi, and M. D. Ajarim, "Reactions of organolithium reagents with quinazoline derivatives," Arkivoc, vol. 2012, no. 7, pp. 35-78, 2012.

[15] K. Smith, G. A. El-Hiti, and S. A. Hegazy, "A simple and convenient procedure for lithiation and side-chain substitution of 2-alkyl-4-(methylthio)quinazolines and 2-alkyl-4methoxyquinazolines," Synthesis, no. 17, pp. 2951-2961, 2005.

[16] G. M. Sheldrick, "Phase annealing in SHELX-90: direct methods for larger structures," Acta Crystallographica Section A: Foundations of Crystallography, vol. 46, no. 6, pp. 467-473, 1990.

[17] G. M. Sheldrick, SHELXS-97, Program for Crystal Structure Refinement, Universität Göttingen, Göttingen, Germany, 1997.

[18] M. A. Palafox, V. K. Rastogi, R. P. Tanwar, and L. Mittal, "Vibrational frequencies and structure of 2-thiouracil by Hartree-Fock, post-Hartree-Fock and density functional methods," Spectrochimica Acta Part A: Molecular and Biomolecular Spectroscopy, vol. 59, no. 11, pp. 2473-2486, 2003.

[19] G. N. Ten, V. V. Nechaev, A. N. Pankratov, V. I. Berezin, and V. I. Baranov, "Effect of hydrogen bonding on the structure and vibrational spectra of the complementary pairs of nucleic acid bases. II. adenine-thymine," Journal of Structural Chemistry, vol. 51, no. 5, pp. 854-861, 2010.

[20] Ç. Çırak and N. Koç, "Molecular structure and effects of intermolecular hydrogen bonding on the vibrational spectrum of trifluorothymine, an antitumor and antiviral agent," Journal of Molecular Modeling, vol. 18, no. 9, pp. 4453-4464, 2012.

[21] M. A. Palafox, G. Tardajos, A. Guerrero-Martínez et al., "FTIR, FT-Raman spectra, density functional computations of the vibrational spectra and molecular geometry of biomolecule 5aminouracil," Chemical Physics, vol. 340, no. 1-3, pp. 17-31, 2007.

[22] J. S. Singh, "FTIR and Raman spectra and fundamental frequencies of biomolecule: 5-methyluracil (thymine)," Journal of Molecular Structure, vol. 876, no. 1-3, pp. 127-133, 2008.

[23] M. H. Jamróz, J. C. Dobrowolski, and R. Brzozowski, "Vibrational modes of 2,6-, 2,7-, and 2,3-diisopropylnaphthalene. A DFT study," Journal of Molecular Structure, vol. 787, no. 1-3, pp. 172-183, 2006.

[24] Ç. Çırak, Y. Sert, and F. Ucun, "Experimental and computational study on molecular structure and vibrational analysis of a modified biomolecule: 5-Bromo-2'-deoxyuridine," Spectrochimica Acta Part A: Molecular and Biomolecular Spectroscopy, vol. 92, no. 6, pp. 406-414, 2012.

[25] Y. Zhao and D. G. Truhlar, "The M06 suite of density functionals for main group thermochemistry, thermochemical kinetics, noncovalent interactions, excited states, and transition elements: two new functionals and systematic testing of four M06-class functionals and 12 other functionals," Theoretical Chemistry Accounts, vol. 120, no. 1-3, pp. 215-241, 2008.

[26] K. Helios, R. Wysokiński, A. Pietraszko, and D. Michalska, "Vibrational spectra and reinvestigation of the crystal structure of a polymeric copper(II)-orotate complex, $\left[\mathrm{Cu}(\mu\right.$-HOr $)\left(\mathrm{H}_{2} \mathrm{O}\right)$ $2] n$ : the performance of new DFT methods, M06 and M05-2X, in theoretical studies," Vibrational Spectroscopy, vol. 55, no. 2, pp. 207-215, 2011.

[27] J. Gu, J. Wang, and J. Leszczynski, "Stacking and H-bonding patterns of dGpdC and dGpdCpdG: performance of the M05$2 \mathrm{X}$ and M06-2X Minnesota density functionals for the single strand DNA," Chemical Physics Letters, vol. 512, no. 1-3, pp.108$112,2011$.

[28] K. H. Lemke and T. M. Seward, "Thermodynamic properties of carbon dioxide clusters by M06-2X and dispersion-corrected 
B2PLYP-D theory," Chemical Physics Letters, vol. 573, no. 6, pp. 19-23, 2013.

[29] E. I. Paulraj and S. Muthu, "Spectroscopic studies (FTIR, FT-Raman and UV), potential energy surface scan, normal coordinate analysis and $\mathrm{NBO}$ analysis of $(2 R, 3 R, 4 R, 5 S)-1-$ (2-hydroxyethyl)-2-(hydroxymethyl) piperidine-3,4,5-triol by DFT methods," Spectrochimica Acta Part A: Molecular and Biomolecular Spectroscopy, vol. 108, no. 5, pp. 38-49, 2013.

[30] U. Yadava, M. Singh, and M. Roychoudhury, "Gas-phase conformational and intramolecular $\pi-\pi$ interaction studies on some pyrazolo[3,4-d]pyrimidine derivatives," Computational and Theoretical Chemistry, vol. 977, no. 1-3, pp. 134-139, 2011.

[31] C. N. Ramachandran and E. Ruckenstein, "Density functional theoretical studies of the isomers of croconic acid and their dimers," Computational and Theoretical Chemistry, vol. 973, no. 1-3, pp. 28-32, 2011.

[32] Y. Sert, Ç. Çırak, and F. Ucun, "Vibrational analysis of 4chloro-3-nitrobenzonitrile by quantum chemical calculations," Spectrochimica Acta Part A: Molecular and Biomolecular Spectroscopy, vol. 107, no. 4, pp. 248-255, 2013.

[33] A. Frish, A. B. Nielsen, and A. J. Holder, Gauss View User Manual, Gaussian Inc, Pittsburg, Pa, USA, 2001.

[34] W. H. James, E. G. Buchanan, C. W. Müller et al., "Evolution of amide stacking in larger $\gamma$-peptides: triamide H-bonded cycles," Journal of Physical Chemistry A, vol. 115, no. 47, pp. 13783-13798, 2011.

[35] M. J. Frisch, G. W. Trucks, H. B. Schlegel et al., Gaussian 03, Revision D.01, Gaussian, Wallingford, Conn, USA, 2004.

[36] M. H. Jamróz, Vibrational Energy Distribution Analysis VEDA 4, 2004.

[37] M. H. Jamróz, "Vibrational energy distribution analysis (VEDA): scopes and limitations," Spectrochimica Acta Part A: Molecular and Biomolecular Spectroscopy, vol. 114, no. 10, pp. 220-230, 2013.

[38] H. Arslan and Ö. Algül, "Vibrational spectrum and assignments of 2-(4-methoxyphenyl)- $1 H$-benzo[d]imidazole by ab initio Hartree-Fock and density functional methods," Spectrochimica Acta Part A: Molecular and Biomolecular Spectroscopy, vol. 70, no. 1, pp. 109-116, 2008.

[39] C. Irak, S. Demir, F. Ucun, and O. Ubuk, "Experimental and theoretical study on the structure and vibrational spectra of $\beta-2$ aminopyridinium dihydrogenphosphate," Spectrochimica Acta Part A: Molecular and Biomolecular Spectroscopy, vol. 79, no. 3, pp. 529-532, 2011.

[40] G. A. El-Hiti, K. Smith, A. S. Hegazy, M. D. Ajarim, and B. M. Kariuki, "Crystal structure of 2-[4-(methylsulfanyl)quinazolin2-yl]-1-phenylethanol," Acta Crystallographica Section E: Crystallographic Communications, vol. 70, no. 11, p. o1101, 2014.

[41] C. Y. Panicker, H. T. Varghese, K. R. Ambujakshan et al., "Vibrational spectra and computational study of 3-amino-2phenyl quinazolin-4(3H)-one," Journal of Molecular Structure, vol. 963, no. 2-3, pp. 137-144, 2010.

[42] C. Y. Panicker, H. T. Varghese, K. R. Ambujakshan et al., "Ab initio and density functional theory studies on vibrational spectra of 3-\{[(4-methoxyphenyl)methylene $]$ amino $\}-2-$ phenylquinazolin-4(3H)-one," European Journal of Chemistry, vol. 1, no. 1, pp. 37-43, 2010.

[43] J. P. Jasinski, J. A. Golen, A. S. Praveen, H. S. Yathirajan, and B. Narayana, "Methyl 3,5-dibromo-2-diacetylaminobenzoate," Acta Crystallographica Section E: Crystallographic Communications, vol. 67, no. 9, p. o2503, 2011.
[44] C.-L. He, Z.-M. Du, Z.-Q. Tang, X.-M. Cong, and L.-Q. Meng, "N-(1-Diacetylamino- $1 H$-tetrazol-5-yl)acetamide," Acta Crystallographica, Section E: Crystallographic Communications, vol. 65, part 8, article o1902, 2009.

[45] G. Socrates, Infrared and Raman Characteristic Group Frequencies: Tables and Charts, John Wiley \& Sons, Chichester, UK, 3rd edition, 2004.

[46] C. Y. Panicker, H. T. Varghese, K. R. Ambujakshan et al., "FT-IR and FT-Raman spectra and $a b$ initio calculations of 3$\{[(2$-hydroxyphenyl) methylene $]$ amino $\}$-2-phenylquinazolin4(3H)-one," Journal of Raman Spectroscopy, vol. 40, no. 9, pp. 1262-1273, 2009.

[47] C. Y. Panicker, H. T. Varghese, K. R. Ambujakshan et al., "FT-IR, FT-Raman and DFT calculations of 3-[(4-fluorophenyl)methylene] amino-2-phenylquinazolin-4(3H)-one," Journal of Raman Spectroscopy, vol. 40, no. 5, pp. 527-536, 2009.

[48] N. P. G. Roeges, A Guide to the Complete Interpretation of Infrared Spectra of Organic Structures, John Wiley \& Sons, New York, NY, USA, 1994.

[49] P. Larkin, Infrared and Raman Spectroscopy; Principles and Spectral Interpretation, Elsevier, Waltham, Mass, USA, 1st edition, 2011.

[50] C. Y. Panicker, H. T. Varghese, M. Nair, K. Raju, M. Laila, and G. M. Warrier, "Vibrational spectroscopic studies and ab initio calculations of 3-nitroacetanilide," Oriental Journal of Chemistry, vol. 25, no. 3, pp. 643-647, 2009.

[51] I. Yalçin, E. Şener, T. Özden, S. Özden, and A. Akin, "Synthesis and microbiological activity of 5-methyl-2-[p-substituted phenyl]benzoxazoles," European Journal of Medicinal Chemistry, vol. 25, no. 8, pp. 705-708, 1990.

[52] R. Saxena, L. D. Kandpal, and G. N. Mathur, "Synthesis and characterization of poly(benzobisthiazole)s derived from halogenated phthalic acid and isophthalic acid," Journal of Polymer Science, Part A: Polymer Chemistry, vol. 40, no. 22, pp. 39593966, 2002.

[53] R. M. Silverstein and F. X. Webster, Spectrometric Identification of Organic Compound, John Wiley \& Sons, Singapore, 6th edition, 2003.

[54] J. M. Engasser and C. Horvath, "Electrostatic effects on the kinetics of bound enzymes," Biochemical Journal, vol. 145, no. 3, pp. 431-435, 1975.

[55] S. Kundoo, A. N. Banerjee, P. Saha, and K. K. Chattopadhyay, "Synthesis of crystalline carbon nitride thin films by electrolysis of methanol-urea solution," Materials Letters, vol. 57, no. 15, pp. 2193-2197, 2003.

[56] J. Coates and R. A. Meyers, Introduction to Infrared Spectrum. A Practical Approach, John Wiley \& Sons, Chichester, UK, 2000.

[57] M. Pagannone, B. Fornari, and G. Mattei, "Molecular structure and orientation of chemisorbed aromatic carboxylic acids: surface enhanced Raman spectrum of benzoic acid adsorbed on silver sol," Spectrochimica Acta Part A: Molecular Spectroscopy, vol. 43, no. 5, pp. 621-625, 1987.

[58] S. R. Kumar, N. Vijay, K. Amarendra, P. Onkar, and S. Leena, "Theoretical studies on the isomers of quinazolinone by first principles," Research Journal of Recent Sciences, vol. 1, no. 3, pp. 11-18, 2012.

[59] D. L. Pavia, G. M. Lampman, and G. S. Kriz, Introduction to Spectroscopy, Harcourt College Publishers, 2001.

[60] E. Kavitha, N. Sundaraganesan, S. Sebastian, and M. Kurt, "Molecular structure, anharmonic vibrational frequencies and $\mathrm{NBO}$ analysis of naphthalene acetic acid by density functional 
theory calculations," Spectrochimica Acta Part A: Molecular and Biomolecular Spectroscopy, vol. 77, no. 3, pp. 612-619, 2010.

[61] K. Chaitanya, "Molecular structure, vibrational spectroscopic (FT-IR, FT-Raman), UV-vis spectra, first order hyperpolarizability, NBO analysis, HOMO and LUMO analysis, thermodynamic properties of benzophenone 2,4-dicarboxylic acid by ab initio HF and density functional method," Spectrochimica Acta-Part A: Molecular and Biomolecular Spectroscopy, vol. 86, pp. 159-173, 2012.

[62] E. Kavitha, N. Sundaraganesan, and S. Sebastian, "Molecular structure, vibrational spectroscopic and HOMO, LUMO studies of 4-nitroaniline by density functional method," Indian Journal of Pure \& Applied Physics, vol. 48, no. 1, pp. 20-30, 2010.

[63] A. Jayaprakash, V. Arjunan, and S. Mohan, "Vibrational spectroscopic, electronic and quantum chemical investigations on 2,3-hexadiene," Spectrochimica Acta Part A: Molecular and Biomolecular Spectroscopy, vol. 81, no. 1, pp. 620-630, 2011.

[64] M. Govindarajan, M. Karabacak, A. Suvitha, and S. Periandy, "FT-IR, FT-Raman, ab initio, HF and DFT studies, NBO, HOMO-LUMO and electronic structure calculations on 4chloro-3-nitrotoluene," Spectrochimica Acta-Part A: Molecular and Biomolecular Spectroscopy, vol. 89, pp. 137-148, 2012. 

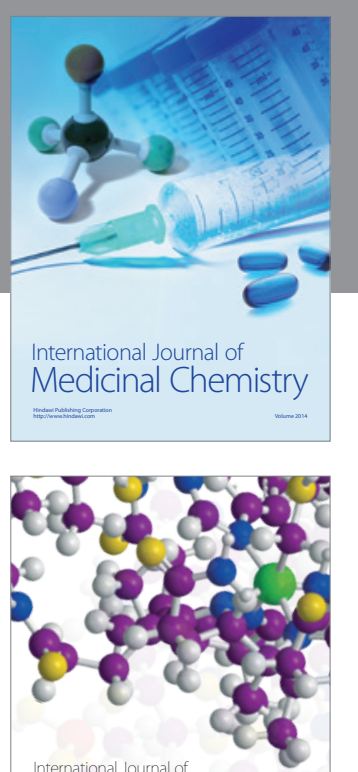

Carbohydrate Chemistry

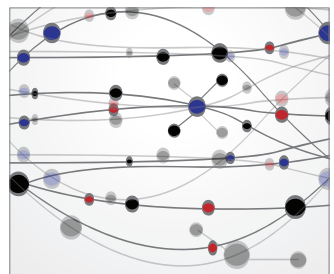

The Scientific World Journal
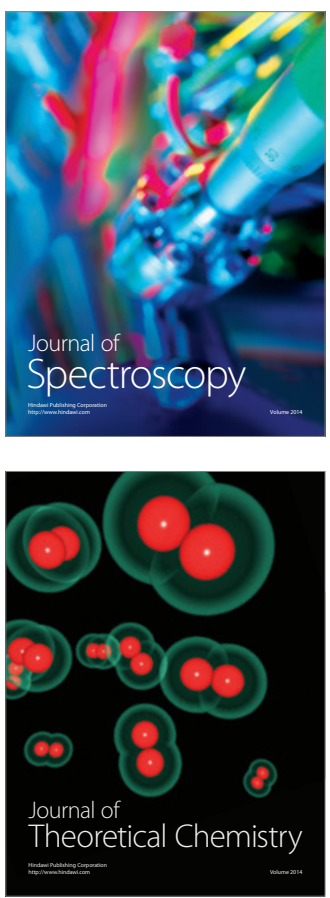
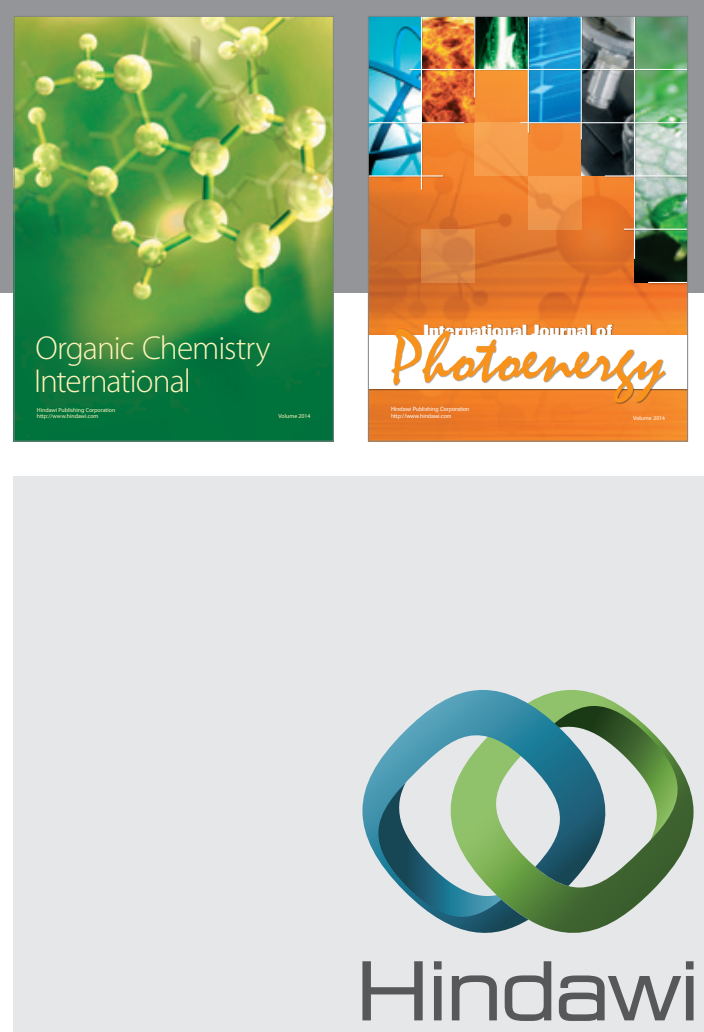

Submit your manuscripts at

http://www.hindawi.com

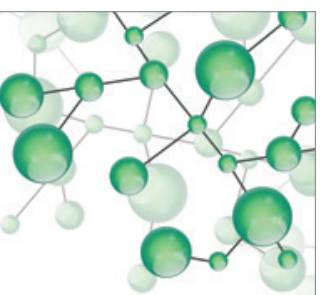

International Journal of

Inorganic Chemistry

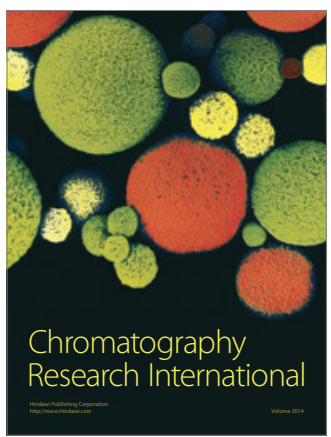

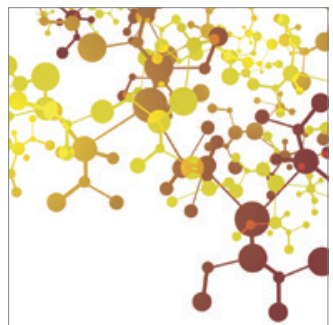

Applied Chemistry
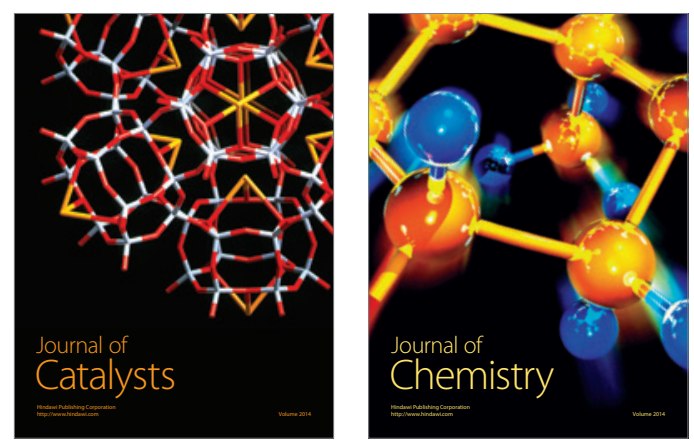
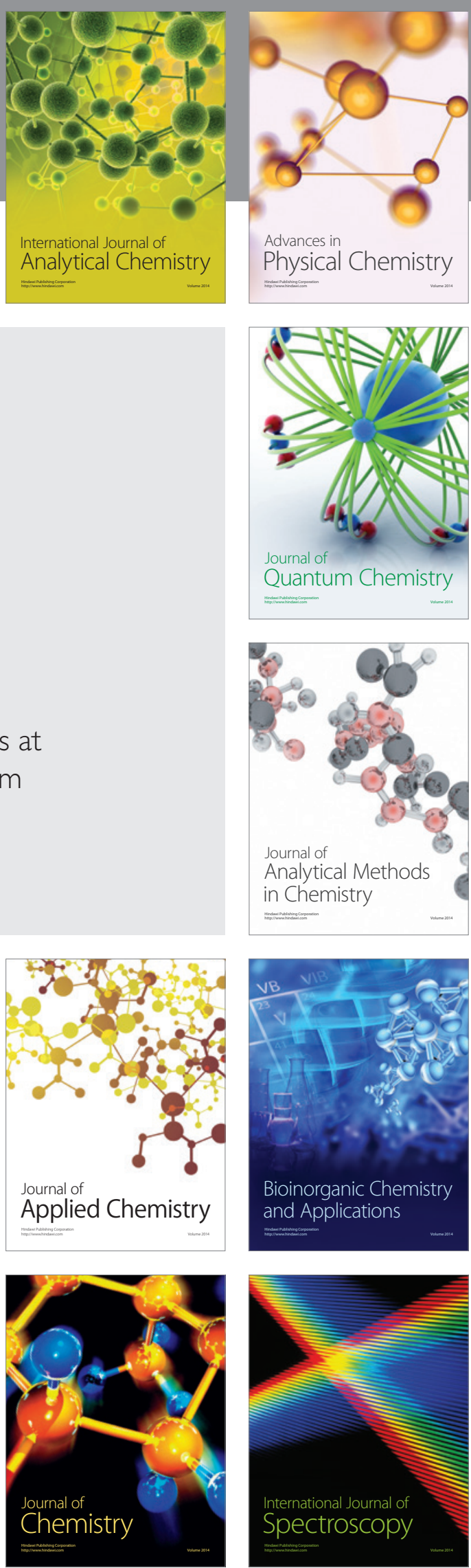